\title{
Paraconvex functions and paraconvex sets
}

\author{
by \\ Huynh Van Ngai (Qui Nhon) and Jean-Paul Penot (Pau)
}

\begin{abstract}
We study a class of functions which contains both convex functions and differentiable functions whose derivatives are locally Lipschitzian or Hölderian. This class is a subclass of the class of approximately convex functions. It enjoys refined properties. We also introduce a class of sets whose associated distance functions are of that type. We discuss the properties of the metric projections on such sets under some assumptions on the geometry of the Banach spaces in which they are embedded. We describe some relations between such sets and functions.
\end{abstract}

1. Introduction. Some classes of sets or functions are so favorable for smooth and nonsmooth analysis that they have been considered by a number of authors. This is the case for the class of (locally) $p$-paraconvex functions introduced in [56] and studied in [3], [5], [8], [9], [10], [12], [15], [30], [45], [51], [56]-[69], especially in the case $p=2$ in which case the functions are also called semiconvex as in [32] and the book [11] which stresses their applications in optimal control and the study of Hamilton-Jacobi equations. This class contains the class of convex functions and those of class $C^{2}$ and enjoys interesting stability properties. In the present paper we study the notion of $p$-paraconvexity for sets introduced in [69] (in a global sense and for $p=2$ ), studied in [15, Section 5] under the name of property $(\omega)$ and called subsmoothness in [5]. We also introduce a notion of intrinsically $p$-paraconvex set. Both notions are given in terms of the distance function to the set. As in the case of the (more general) classes of approximately convex functions and sets in the sense of [5], [15], [18], [38], [39], [40] which differs from the one used in [24], [25], we provide characterizations. It turns out that the classes of $p$-paraconvex functions and sets form more structured classes in which one has a quantitative control of the variations of the subdifferentials of the functions or of the normal cone to the set at nearby points respectively. For instance, in the class of superreflexive Banach spaces, we show

2000 Mathematics Subject Classification: 49J52, 46N10, 46T20.

Key words and phrases: approximately convex function, approximately convex set, monotonicity, nonsmooth analysis, normal, projection, subdifferential. 
that best approximations to $p$-paraconvex sets exist and we prove the Hölder continuity of projections, obtaining results somewhat similar to the case of convex subsets (see [1], [2], [50] and their references). We also present conditions ensuring that a marginal function is p-paraconvex (see Section 4), thus solving an unsettled problem. We relate the concepts of paraconvexity for sets and functions through epigraphs and sublevel sets in Section 7. Let us add that for the class of $p$-paraconvex functions (resp. sets) the usual notions of subdifferential (resp. normal) coincide; this fact makes these classes particularly attractive.

The concept of $p$-paraconvexity around a point we study is a localization of a notion introduced in [56]; let us note that the version we study is not global as in [10], [56]-[62], [69]. However, it amounts to $p$-paraconvexity in the sense of [56] on some neighborhood of the point. Since it has been proved in [56] that for $p>2$ a $p$-paraconvex function whose restrictions to line segments are absolutely continuous is convex, in the following we restrict our attention to the case $p \in[1,2]$.

Definition 1. Given some $p \in[1,2]$, a function $f: X \rightarrow \mathbb{R}_{\infty}:=$ $\mathbb{R} \cup\{+\infty\}$ on a normed vector space $X$ is said to be $p$-paraconvex around $\bar{x} \in \operatorname{dom} f:=f^{-1}(\mathbb{R})$ if there exist $c, \delta>0$ such that for any $x, y \in B(\bar{x}, \delta)$ and any $t \in[0,1]$,

$$
f(t x+(1-t) y) \leq t f(x)+(1-t) f(y)+c t(1-t)\|x-y\|^{p} .
$$

The function $f$ is said to be (locally) p-paraconvex on an open subset $U$ of $X$ if it is finite and $p$-paraconvex around each point of $U$. The index of $p$-paraconvexity of $f$ around $\bar{x}$ is the infimum $c_{f}$ of the set of real numbers $c$ such that there exists some $\delta>0$ for which relation (1) is satisfied for any $x, y \in B(\bar{x}, \delta)$ and $t \in[0,1]$.

When $c_{f}$ is negative, we have in fact a strong convexity property; the case $c_{f}=0, p=1$ corresponds to approximate convexity (see [38], [39]). The case $p=2$ is of special interest, in particular in the setting of Euclidean spaces or Hilbert spaces. As mentioned above, it has been thoroughly studied, at least in finite dimensions, in Hilbert spaces, and in the locally Lipschitzian case. Here we get rid of these restrictions. We relate the notion of $p$-paraconvexity to the following notion of $p$-paramonotonicity. For $p=2$ it corresponds to a localized version of a concept introduced by A. Pazy [42] (see also [17], [43] for related notions).

Definition 2. A multimap (or set-valued map) $M: X \rightrightarrows X^{*}$ is said to be $p$-paramonotone around $\bar{x}$ on a subset $E$ of $X$ if there exist some $m, \delta>0$ such that for any $x_{1}, x_{2} \in E \cap B(\bar{x}, \delta), x_{1}^{*} \in M\left(x_{1}\right)$ and $x_{2}^{*} \in M\left(x_{2}\right)$,

$$
\left\langle x_{1}^{*}-x_{2}^{*}, x_{1}-x_{2}\right\rangle \geq-m\left\|x_{1}-x_{2}\right\|^{p} .
$$


For $E=X$ one simply says that $M$ is $p$-paramonotone around $\bar{x}$. The index of p-paramonotonicity of $M$ on $E$ around $\bar{x}$ is the infimum $m_{M}$ of the set of real numbers $m>0$ such that there exists some $\delta>0$ for which relation (2) is satisfied for any $x_{1}, x_{2} \in E \cap B(\bar{x}, \delta), x_{1}^{*} \in M\left(x_{1}\right)$ and $x_{2}^{*} \in M\left(x_{2}\right)$.

Directional versions of the concepts we study can be introduced, but for brevity we do not consider them here. We also leave apart the generalization of $p$-paraconvexity consisting in replacing the term $c t(1-t)\|x-y\|^{p}$ in (1) by $c t(1-t) \alpha(\|x-y\|)$ where $\alpha: \mathbb{R}_{+} \rightarrow \mathbb{R}_{+} \cup\{+\infty\}$ is a hypermodulus, i.e. a nondecreasing function such that $\alpha(t) / t \rightarrow 0$ as $t \rightarrow 0_{+}$. The reason for that choice lies in the Hölder estimates we get for the negative of the distance function to an arbitrary closed subset of a uniformly smooth Banach space (Corollary 13) and for the metric projection onto a $p$-paraconvex subset of a superreflexive Banach space (Theorem 30); this last topic has been thoroughly studied for convex subsets (see [1], [2] for instance). The notion of $p$-paraconvex subset is studied in Sections 5 and 6 . Although the choice $p=2$ is the most important one, the case $p \neq 2$ may be of interest, for instance when working in some $L_{p}$ space, as illustrated by Proposition 24 below.

2. Preliminaries. In the following, $X$ is a Banach space with topological dual space $X^{*}$ and $\mathcal{S}(X)$ denotes the set of lower semicontinuous (l.s.c.) functions $f: X \rightarrow \mathbb{R}_{\infty}:=\mathbb{R} \cup\{+\infty\}$. The open ball with center $\bar{x} \in X$ and radius $\varrho>0$ is denoted by $B\left(\bar{x}, \varrho\right.$ ), while $\bar{B}_{X}$ (resp. $\bar{B}_{X^{*}}$ ) stands for the closed unit ball of $X$ (resp. $X^{*}$ ) and $S_{X}$ stands for the unit sphere. Given a subset $E$ of $X$, the distance function $d_{E}$ associated with $E$ is given by $d_{E}(x):=\inf _{e \in E} d(x, e)$. We write $\left(x_{n}\right) \stackrel{E}{\rightarrow} x$ to mean that $\left(x_{n}\right) \rightarrow x$ and $x_{n} \in E$ for each $n \in \mathbb{N}$.

Since the functions we consider are not necessarily smooth, we have to use some generalizations of derivatives. A first possibility is to take a primal approach by considering directional derivatives. A second approach is a dual one; it is a one-sided substitute to the derivative called a subdifferential. Several sets of axioms have been devised for such a concept. The notion of subdifferential we adopt here is as versatile as possible: a subdifferential on a Banach space $X$ will just be a correspondence $\partial: \mathcal{F}(X) \times X \rightrightarrows X^{*}$ which assigns a subset $\partial f(x)$ of the dual space $X^{*}$ of $X$ to any $f$ in a subclass $\mathcal{F}(X)$ of the set $\mathcal{S}(X)$ of lower semicontinuous functions on $X$ and any $x \in X$ at which $f$ is finite; we assume it has the following natural property:

(M) If $f \in \mathcal{F}(X)$ is a Lipschitzian function and if $\bar{x}$ is a minimizer of $f$, then $0 \in \partial f(\bar{x})$.

Of course, usual subdifferentials also satisfy other conditions which turn them into calculus properties. These include a form of the mean value the- 
orem we describe below. Another property is the nonemptiness of $\partial f(x)$ for $x$ in a dense subset of $X$ for any Lipschitzian function $f$ on $X$; then we say that $X$ is a (Lipschitz) $\partial$-subdifferentiability space. This property is satisfied if $X$ is an Asplund space and $\partial$ is larger than the Fréchet subdifferential (sometimes called the firm subdifferential) defined by

$x^{*} \in \partial^{F} f(x) \Leftrightarrow \forall \varepsilon>0 \exists \delta>0, \forall u \in \delta \bar{B}_{X}: f(x+u) \geq f(x)+\left\langle x^{*}, u\right\rangle-\varepsilon\|u\|$.

If $X$ is smooth enough, for instance if $X$ is a Hilbert space, and if $p \in] 1,2]$, this property is satisfied by the $p$-proximal subdifferential $\partial^{p}$; recall that for $p \in(1,+\infty)$, the $p$-proximal subdifferential of a function $f$ on a n.v.s. $X$ at $x \in X$ is given by

$x^{*} \in \partial^{p} f(x) \Leftrightarrow \exists c>0, \varrho>0, \forall u \in \varrho \bar{B}_{X}: f(x+u) \geq f(x)+\left\langle x^{*}, u\right\rangle-c\|u\|^{p}$.

Clearly $\partial^{p} f(x)$ is contained in the Fréchet subdifferential $\partial^{F} f(x)$. Albeit property (M) is satisfied for $\partial^{p}$ for any $p>1$, the practical use of $\partial^{p}$ seems to be limited to the case $p \in] 1,2]$.

With any subdifferential $\partial$ is associated a notion of normal cone obtained by setting, for a subset $E$ of $X$ and $e \in E$,

$$
N(E, e):=\mathbb{R}_{+} \partial \iota_{E}(e)
$$

where $\iota_{E}$ is the indicator function of $E$, given by $\iota_{E}(x)=0$ if $x \in E,+\infty$ otherwise. In the cases $\partial=\partial^{D}, \partial=\partial^{C}$ and $\partial=\partial^{F}$ we get the normal cones $N^{D}(E, e), N^{C}(E, e)$ and $N^{F}(E, e)$ to $E$ at $e \in E$ in the senses of Bouligand, Clarke and Fréchet respectively, as defined below. Other notions have been devised by Ioffe [26], [27], Michel-Penot [36], Mordukhovich [37], Treiman [68] and several other authors.

Conversely, to a notion $N$ of normal cone one can associate a notion of subdifferential $\partial$ by setting $E_{f}:=\{(x, r) \in X \times \mathbb{R}: r \geq f(x)\}, x_{f}:=$ $(x, f(x))$ and

$$
\partial f(x):=\left\{x^{*} \in X^{*}:\left(x^{*},-1\right) \in N\left(E_{f}, x_{f}\right)\right\} .
$$

Now let us give the examples of those concepts we will use most. The (contingent or directional or Dini-Hadamard) normal cone $N^{D}(E, x)$ to a subset $E$ of $X$ at $x \in \operatorname{cl}(E)$ is the polar cone of the directional (or DiniHadamard or Bouligand) tangent cone $T^{D}(E, x)$ to $E$ at $x$, which is the set of vectors $v \in X$ such that there exist sequences $\left(t_{n}\right) \rightarrow 0_{+}$and $\left(x_{n}\right) \stackrel{E}{\rightarrow} x$ for which $\left(t_{n}^{-1}\left(x_{n}-x\right)\right) \rightarrow v$. Both cones play a crucial role in nonlinear analysis and optimization.

The Fréchet normal cone (or firm normal cone) to $E$ at $x$ is given by $x^{*} \in N^{F}(E, x) \Leftrightarrow \forall \varepsilon>0 \exists \delta>0, \forall u \in E \cap B(x, \delta):\left\langle x^{*}, u-x\right\rangle \leq \varepsilon\|u-x\|$. The Clarke normal cone $N^{C}(E, x)$ to a subset $E$ of $X$ at $x \in \operatorname{cl}(E)$ is the polar cone of the Clarke tangent cone $T^{C}(E, x)$ to $E$ at $x$, which is the set 
of vectors $v \in X$ such that for any sequence $\left(x_{n}\right) \stackrel{E}{\rightarrow} x$ there exist sequences $\left(t_{n}\right) \rightarrow 0_{+}$and $\left(y_{n}\right)$ in $E$ for which $\left(t_{n}^{-1}\left(y_{n}-x_{n}\right)\right) \rightarrow v$.

The (lower) directional derivative (or contingent derivative or lower DiniHadamard derivative) of $f$ at $x$ is given by

$$
f^{D}(x, v):=\liminf _{(t, w) \rightarrow\left(0_{+}, v\right)} \frac{f(x+t w)-f(x)}{t} .
$$

The Clarke-Rockafellar derivative or circa-derivative of a function $f: X \rightarrow$ $\overline{\mathbb{R}}=\mathbb{R} \cup\{-\infty,+\infty\}$ finite at $x$ is given by the following formula:

$$
f^{C}(x, v):=\inf _{r>0} \limsup _{\substack{f(y) \rightarrow f(x) \\(t, y) \rightarrow\left(0_{+}, x\right)}} \inf _{w \in B(v, r)} \frac{f(y+t w)-f(y)}{t} .
$$

Both derivatives are majorized by the dag derivative which is given by

$$
f^{\dagger}(x, v):=\limsup _{(t, y) \rightarrow\left(0_{+}, x\right), f(y) \rightarrow f(x)} \frac{f(y+t(v+x-y))-f(y)}{t} .
$$

The interest of such a derivative seems to be limited to questions of generalized convexity and to the fact that the associated subdifferential is large. Here, the subdifferential associated with some generalized derivative $f^{\prime}$ is defined by

$$
\partial f(x):=\left\{x^{*} \in X^{*}: x^{*}(\cdot) \leq f^{\prime}(x, \cdot)\right\} .
$$

For $f^{\prime}=f^{D}$ (resp. $f^{\prime}=f^{C}$ ) one recovers the Bouligand or Dini-Hadamard subdifferential $\partial^{D}$ (resp. Clarke subdifferential $\partial^{C}$ ).

Given a subdifferential $\partial$, following [31], [37], [47], one can associate to it a corresponding limiting subdifferential $\bar{\partial}$ by setting, for a l.s.c. function $f$ and a point $x$ of its domain,

$$
\bar{\partial} f(x):=w^{*}-\limsup _{(u, f(u)) \rightarrow(x, f(x))} \partial f(u),
$$

where the $w^{*}$-limsup is the set of weak ${ }^{*}$ cluster points of bounded nets $\left(u_{i}^{*}\right)_{i \in I}$ with $u_{i}^{*} \in \partial f\left(u_{i}\right),\left(u_{i}\right)_{i \in I} \rightarrow x$ and $\left(f\left(u_{i}\right)\right)_{i \in I} \rightarrow f(x)$.

We will make use of mean value inequalities. Under a primal form, such estimates have been pointed out in [44]. In a dual form, as used here, they have been devised by Zagrodny for the Clarke subdifferential [71], [72], and by Loewen [34] for the Fréchet subdifferential. In [49], such a result is put in a general form including the Ioffe subdifferential [26], [27], the moderate subdifferential [36], and, in Asplund spaces, the Fréchet subdifferential, the Hadamard subdifferential and the limiting subdifferential. In [4] such a property is obtained under different assumptions.

Definition 3 (Fuzzy mean value property). A subdifferential $\partial$ is said to be valuable on $X$ if for any $\bar{x} \in X$ and $\bar{y} \in X \backslash\{\bar{x}\}$, any lower semicontinuous function $f: X \rightarrow \mathbb{R} \cup\{+\infty\}$ finite at $\bar{x} \in X$, and any $r \in \mathbb{R}$ such 
that $f(\bar{y}) \geq r$, there exist $u \in[\bar{x}, \bar{y})$ and sequences $\left(u_{n}\right) \rightarrow u$ and $\left(u_{n}^{*}\right)$ such that $u_{n}^{*} \in \partial f\left(u_{n}\right),\left(f\left(u_{n}\right)\right) \rightarrow f(u)$, and

(3) $\quad \liminf _{n}\left\langle u_{n}^{*}, \bar{y}-\bar{x}\right\rangle \geq r-f(\bar{x})$,

(4) $\quad \liminf _{n}\left\langle u_{n}^{*}, x-u_{n}\right\rangle \geq \frac{\|x-u\|}{\|\bar{y}-\bar{x}\|}(r-f(\bar{x}))$

$$
\forall x \in\left(\bar{x}+\mathbb{R}_{+}(\bar{y}-\bar{x})\right) \backslash[\bar{x}, u),
$$

(5) $\quad \lim _{n}\left\|u_{n}^{*}\right\| d\left(u_{n},[\bar{x}, \bar{y}]\right)=0$.

A simpler version due to Lebourg [33] is as follows. It is satisfied for $\partial:=\partial^{C}$, the Clarke subdifferential, the moderate subdifferential $\partial^{M}$ of [36], and if $X$ is Asplund, by the Bouligand, Fréchet and limiting subdifferentials.

Definition 4. A subdifferential $\partial$ is said to be Lipschitz-valuable on $X$ if for any $\bar{x}, \bar{y} \in X$ and any Lipschitzian function $f: X \rightarrow \mathbb{R}$ one can find $w \in[\bar{x}, \bar{y}]$ and $w^{*} \in \partial f(w)$ such that $f(y)-f(x)=\left\langle w^{*}, y-x\right\rangle$.

We need some results about subdifferentials of distance functions.

LEMma 5 ([40], [48]). Let $E$ be a nonempty subset of a Banach space $X$ and let $x \in E, v \in X$. Then

$$
d_{E}^{C}(x, v)=\limsup _{t \rightarrow 0_{+}, e \stackrel{E}{\rightarrow} x} \frac{1}{t} d_{E}(e+t v) .
$$

The next result is close to [7, Lemma 6], [27, Lemma 5], [48, Lemma 1], [41, Lemma 3.6] and [5, Lemma 3.7], but it contains a crucial additional information obtained in [40]. Recall that the norm of $X$ is said to satisfy the Kadec-Klee property if for every $x \in X$, a sequence $\left(x_{n}\right)$ converges to $x$ whenever it weakly converges to $x$ and $\left(\left\|x_{n}\right\|\right) \rightarrow\|x\|$.

LEMMA 6. Suppose that $E$ is a closed nonempty subset of an Asplund space $X, w \in X \backslash E$ and $w^{*} \in \partial^{F} d_{E}(w)$. Then $\left\|w^{*}\right\|=1$ and there exist sequences $\left(x_{n}\right),\left(x_{n}^{*}\right)$ in $E$ and $X^{*}$ respectively such that $x_{n}^{*} \in \partial^{F} d_{E}\left(x_{n}\right)$ for each $n \in \mathbb{N}$ and

$$
\left(\left\|x_{n}-w\right\|\right) \rightarrow d_{E}(w), \quad\left(\left\langle x_{n}^{*}, w-x_{n}\right\rangle\right) \rightarrow d_{E}(w), \quad\left(\left\|x_{n}^{*}-w^{*}\right\|\right) \rightarrow 0 .
$$

If moreover $X$ is reflexive and if its norm has the Kadec-Klee property, then a subsequence of $\left(x_{n}\right)$ converges to some best approximation $x$ of $w$ in $E$ and $\left\langle w^{*}, w-x\right\rangle=\|x-w\|=d_{E}(w)$.

Now consider the limiting normal cone

$$
\bar{N}(E, a):=\limsup _{x \stackrel{E}{\rightarrow} a} N(E, x)
$$


and the limiting Fréchet normal cone

$$
\overline{N^{F}}(E, a):=\limsup _{x \stackrel{E}{\rightarrow} a} N^{F}(E, x)
$$

obtained from the normal cone (resp. the Fréchet normal cone) by the weak upper limit process described above.

Corollary 7 ([40]). Let $E$ be a closed nonempty subset of an Asplund space $X$ and let $\bar{x}^{*} \in \overline{\partial^{F}} d_{E}(\bar{x})$ with $\bar{x} \in E$. Then $\left\|\bar{x}^{*}\right\| \leq 1$ and there exist sequences $\left(x_{n}\right),\left(x_{n}^{*}\right)$ in $E$ and $X^{*}$ respectively such that $\left(x_{n}\right) \rightarrow \bar{x},\left(x_{n}^{*}\right) \rightarrow \bar{x}^{*}$ weak* and $x_{n}^{*} \in \partial^{F} d_{E}\left(x_{n}\right)$ for each $n \in \mathbb{N}$.

3. Paraconvexity of functions. The following characterization of $p$ paraconvexity has been given in $[14$, Thm. 5.1] in the case where $p=2, X$ is finite-dimensional and the function is Lipschitzian; there, only the proximal subdifferential is used and equivalence with the lower- $\mathrm{C}^{2}$ property in the sense of [54], [65] is proved. This characterization is extended to the case where $X$ is a Hilbert space and the function is lower semicontinuous in $[5$, Thm. 4.1], using the Clarke-Rockafellar subdifferential. Here we consider the case of a general Banach space and of an arbitrary valuable subdifferential; the argument for the implication $(d) \Rightarrow(a)$ is different.

TheOREM 8. Let $p \in] 1,2]$, let $f \in \mathcal{F}(X)$ and let $\bar{x} \in \operatorname{dom} f$. Suppose the subdifferential $\partial f$ of $f$ is contained in $\partial^{\dagger} f$. Then $(\mathrm{a}) \Rightarrow(\mathrm{b}) \Rightarrow(\mathrm{c}) \Rightarrow(\mathrm{d})$ below. If moreover $\partial$ is valuable, all these assertions are equivalent.

(a) $f$ is p-paraconvex around $\bar{x}$;

(b) there exist $c \in \mathbb{R}$ and $\varrho>0$ such that for any $x \in B(\bar{x}, \varrho) \cap \operatorname{dom} f$ and $v \in B(0, \varrho)$,

$$
f^{\dagger}(x, v) \leq f(x+v)-f(x)+c\|v\|^{p}
$$

(c) there exist $c \in \mathbb{R}$ and $\varrho>0$ such that for any $x \in B(\bar{x}, \varrho), x^{*} \in \partial f(x)$ and $v \in B(0, \varrho)$,

$$
\left\langle x^{*}, v\right\rangle \leq f(x+v)-f(x)+c\|v\|^{p}
$$

(d) $\partial f$ is p-paramonotone around $\bar{x}$.

Proof. $(\mathrm{a}) \Rightarrow(\mathrm{b})$. Given $c>c_{f}$, let $\delta>0$ be such that for any $y, z \in$ $B(\bar{x}, \delta)$ and $t \in] 0,1[$,

$$
f((1-t) y+t z) \leq(1-t) f(y)+t f(z)+c t(1-t)\|y-z\|^{p} .
$$

Set $\varrho:=\delta / 2$, fix $x \in B(\bar{x}, \varrho) \cap \operatorname{dom} f$ and take $v \in B(0, \varrho)$ and $y \in B(x, \varrho) \cap$ $\operatorname{dom} f$. Then $z:=x+v \in B(\bar{x}, \delta)$ and

$$
\frac{f((1-t) y+t(x+v))-f(y)}{t} \leq f(x+v)-f(y)+c(1-t)\|y-(x+v)\|^{p},
$$


so that, since $\limsup _{y \rightarrow x}-f(y) \leq-f(x)$,

$$
f^{\dagger}(x, v) \leq f(x+v)-f(x)+c\|v\|^{p} .
$$

(b) $\Rightarrow(\mathrm{c})$ is an immediate consequence of the inclusion $\partial f \subset \partial^{\dagger} f$ and of the definition of $\partial^{\dagger} f$.

$(c) \Rightarrow(d)$ is proved in $[18$, Thm. 2] and [5, Thm. 4.5] for the Clarke subdifferential. The general case is similar: given $c \in \mathbb{R}$ and $\varrho$ as in (c), for any $x, y \in B(\bar{x}, \varrho / 2), x^{*} \in \partial f(x)$ and $y^{*} \in \partial f(y)$, taking $v:=y-x$ and $v^{\prime}:=-v$ we have $x, y \in \operatorname{dom} f$ and

$$
\begin{aligned}
& \left\langle x^{*}, y-x\right\rangle \leq f(y)-f(x)+c\|x-y\|^{p}, \\
& \left\langle y^{*}, x-y\right\rangle \leq f(x)-f(y)+c\|x-y\|^{p},
\end{aligned}
$$

hence $\left\langle x^{*}-y^{*}, x-y\right\rangle \geq-2 c\|x-y\|^{p}$.

$(\mathrm{d}) \Rightarrow(\mathrm{a})$ when $\partial$ is valuable. Assume $\partial f$ is $p$-paramonotone around $\bar{x}$, that is, there exist $\varrho, m>0$ such that for any $x, y \in B(\bar{x}, \varrho), x^{*} \in \partial f(x)$ and $y^{*} \in \partial f(y)$,

$$
\left\langle x^{*}-y^{*}, x-y\right\rangle \geq-m\|x-y\|^{p} .
$$

Let $\delta:=\varrho / 2, x, y \in B(\bar{x}, \delta), t \in] 0,1[, z:=t x+(1-t) y$ and $r<f(z)$. Without loss of generality, to prove (1) we may assume that $x \neq y$ and $f(x), f(y)<+\infty$. Since $\partial$ is valuable we can find $u \in[y, z)$ and sequences $\left(u_{n}\right) \rightarrow u$ and $\left(u_{n}^{*}\right)$ in $X^{*}$ such that $u_{n}^{*} \in \partial f\left(u_{n}\right)$ for each $n$ and

$$
\liminf _{n}\left\langle u_{n}^{*}, \frac{x-u_{n}}{\left\|x-u_{n}\right\|}\right\rangle>\frac{r-f(y)}{\|z-y\|} .
$$

Let $s \in] 0,1\left[\right.$ be such that $z=x+s(u-x)$ and set $z_{n}=x+s\left(u_{n}-x\right)$. Since $\left(u_{n}\right) \rightarrow u$, one has $\left(z_{n}\right) \rightarrow z$, and since $f$ is l.s.c. at $z$, for $n$ larger than a certain $n_{0} \in \mathbb{N}$ one has $u_{n}, z_{n} \in B(\bar{x}, \varrho)$ and $r<f\left(z_{n}\right)$. Moreover $\left\|z_{n}-x\right\|=\left(1-t_{n}\right)\|y-x\|$ for some sequence $\left(t_{n}\right) \rightarrow t$. Using again the fact that $\partial$ is valuable, we obtain $v_{n} \in\left[x, z_{n}\right)$ and sequences $\left(v_{n, k}\right) \rightarrow v_{n}$ and $\left(v_{n, k}^{*}\right)$ such that $v_{n, k} \in B(\bar{x}, \varrho)$ and $v_{n, k}^{*} \in \partial f\left(v_{n, k}\right)$ for any $n \geq n_{0}$ and $k \in \mathbb{N}$, and

$$
\liminf _{k}\left\langle v_{n, k}^{*}, \frac{u_{n}-v_{n, k}}{\left\|u_{n}-v_{n, k}\right\|}\right\rangle>\frac{r-f(x)}{\left\|z_{n}-x\right\|}=\frac{r-f(x)}{\left(1-t_{n}\right)\|y-x\|} .
$$

From (8) there exists some $n_{1} \geq n_{0}$ such that for $n \geq n_{1}$, since $x, z_{n}, u_{n}$ are aligned,

$$
\left\langle u_{n}^{*}, \frac{v_{n}-u_{n}}{\left\|v_{n}-u_{n}\right\|}\right\rangle=\left\langle u_{n}^{*}, \frac{x-u_{n}}{\left\|x-u_{n}\right\|}\right\rangle>\frac{r-f(y)}{\|y-z\|}=\frac{r-f(y)}{t\|y-x\|} .
$$

On the other hand, since $\left(v_{n, k}\right) \rightarrow v_{n}$ for each $n$, by (10) and (9), for each 
$n \geq n_{1}$ one can find $q(n)$ such that for $k \geq q(n)$,

$$
\begin{gathered}
\left\langle u_{n}^{*}, \frac{v_{n, k}-u_{n}}{\left\|v_{n, k}-u_{n}\right\|}\right\rangle>\frac{r-f(y)}{t\|y-x\|}, \\
\left\langle v_{n, k}^{*}, \frac{u_{n}-v_{n, k}}{\left\|u_{n}-v_{n, k}\right\|}\right\rangle>\frac{r-f(x)}{\left(1-t_{n}\right)\|y-x\|} .
\end{gathered}
$$

Adding these inequalities and using (7), we get

$$
m\left\|u_{n}-v_{n, k}\right\|^{p-1} \geq \frac{r-f(y)}{t\|y-x\|}+\frac{r-f(x)}{\left(1-t_{n}\right)\|y-x\|} .
$$

Passing to the limit as $k, n \rightarrow \infty$, we get

$$
m t(1-t)\|y-x\|^{p} \geq(1-t)(r-f(y))+t(r-f(x)) .
$$

Since $r$ is arbitrarily close to $f(z)$, we obtain (1).

Corollary 9. Suppose $f \in \mathcal{F}(X)$ is finite at $\bar{x} \in X$ and p-paraconvex around $\bar{x}$ with $p \in] 1,2]$. Then, for any subdifferential $\partial$ such that $\partial^{p} f \subset$ $\partial f \subset \partial^{\dagger} f$ and for $x$ near $\bar{x}$, one has $\partial f(x)=\partial^{p} f(x)=\partial^{\dagger} f(x)$; in particular, $\partial^{F} f(x)=\partial^{C} f(x)$.

REMARK. The proof we have given yields a quantitative approach: the infimum of the constants $c$ appearing in assertions (b) and (c) is majorized by $c_{f}$ and the index of $p$-paramonotonicity of $\partial f$ around $\bar{x}$ is majorized by $2 c_{f}$. On the other hand, the implication $(\mathrm{d}) \Rightarrow(\mathrm{a})$ shows that $c_{f}$ is majorized by the index of $p$-paramonotonicity of $\partial f$ around $\bar{x}$. We refer to [73] for such estimates in the case of uniformly convex functions which may serve as more precise models.

Corollary 10. Suppose $f: U \rightarrow \mathbb{R}$ is a differentiable function on some open subset $U$ of $X$ with a locally $(p-1)$-Hölderian derivative, with $p \in] 1,2]$. Then $f$ is locally p-paraconvex on $U$.

Proof. Since $f$ is of class $C^{1}$, its Clarke subdifferential is just the derivative $D f$ of $f$. Moreover, $D f$ is locally $p$-paramonotone: for every $\bar{x} \in U$, there exists some $m>0$ and some neighborhood $V$ of $\bar{x}$ contained in $U$ such that for $x, y \in V$,

$$
\langle D f(x)-D f(y), x-y\rangle \geq-m\|x-y\|^{p-1}\|x-y\| .
$$

Thus the result follows from the implication $(\mathrm{d}) \Rightarrow(\mathrm{a})$ of Theorem 8 .

4. Paraconvexity of marginal functions. The statements of the following criteria for $p$-paraconvexity are simpler than the ones in [39] but they rely on differentiability assumptions. We use the following notation. Given an arbitrary set $S$, an open subset $X_{0}$ of a n.v.s. $X, \bar{x} \in X_{0}$ and 
$g: S \times X_{0} \rightarrow \mathbb{R}$, let $f: X_{0} \rightarrow \overline{\mathbb{R}}$ be the marginal function

$$
f(x):=\sup _{s \in S} g(s, x), \quad x \in X_{0} .
$$

We assume $f$ is finite at $\bar{x}$, and for $x \in X_{0}$ and $\varepsilon>0$ we set

$$
\begin{aligned}
& S(x, \varepsilon):= \begin{cases}\{s \in S: g(s, x) \geq f(x)-\varepsilon\} & \text { if } f(x)<+\infty, \\
\left\{s \in S: g(s, x) \geq \varepsilon^{-1}\right\} & \text { if } f(x)=+\infty,\end{cases} \\
& T(x, \varepsilon):=\{s \in S: g(s, x) \geq f(\bar{x})-\varepsilon\} .
\end{aligned}
$$

THEOREM 11. If the following conditions are satisfied for some $m \geq 0$, $p \in] 1,2], \varrho>0$, then the marginal function $f$ is Lipschitzian and $p$ paraconvex on $B(\bar{x}, \delta)$ for some $\delta \in] 0, \varrho[$ :

$\left(C_{1}\right)$ for each $s \in S$ the function $g_{s}:=g(s, \cdot)$ is Fréchet differentiable on the ball $B(\bar{x}, \varrho)$;

$\left(C_{2}\right)$ for every $x \in B(\bar{x}, \varrho)$ one can find $\gamma:=\gamma_{x}>0$ with $\left\|g_{s}^{\prime}(x)\right\| \leq m$ for each $s \in S(x, \gamma)$;

$\left(C_{3}\right)$ there exist $\sigma, \eta>0$ such that $\left\|g_{s}^{\prime}(x)-g_{s}^{\prime}\left(x^{\prime}\right)\right\| \leq \sigma\left\|x-x^{\prime}\right\|^{p-1}$ for any $x, x^{\prime} \in B(\bar{x}, \varrho)$ and $s \in T(x, \eta) \cap T\left(x^{\prime}, \eta\right)$.

Proof. Let us apply the characterization of Theorem 8: we want to find $\beta, \sigma>0$ such that

$$
\forall x \in B(\bar{x}, \beta), \forall v \in \beta \bar{B}_{X}: \quad f^{C}(x, v) \leq f(x+v)-f(x)+\sigma\|v\|^{p} .
$$

Note that $f$ is l.s.c. on $B(\bar{x}, \varrho)$; we will first show that $f$ is Lipschitzian with constant $\ell:=m+\varrho^{p-1} \sigma$ on some ball $B(\bar{x}, 2 \delta)$, where $\sigma, \eta$ are as in $\left(C_{3}\right)$. Let $\varepsilon \in] 0,1[$ be such that $(\ell+2) \varepsilon<\eta$ and let $\delta \in] 0, \varrho / 4[\cap] 0, \varepsilon / 4[$ be such that $f(w)>f(\bar{x})-\varepsilon$ for each $w \in B(\bar{x}, 2 \delta)$. Given $x, y \in B(\bar{x}, 2 \delta)$, we pick $\left.\gamma:=\gamma_{y} \in\right] 0, \varepsilon\left[\operatorname{such}\right.$ that $\left\|g_{s}^{\prime}(y)\right\| \leq m$ for each $s \in S(y, \gamma)$, as in $\left(C_{2}\right)$. Given $s \in S(y, \gamma)$ let $h:[0,1] \rightarrow \mathbb{R}$ and $\bar{t} \in[0,1]$ be defined by $h(t):=$ $g(s, y+t(x-y))$ and

$$
\bar{t}:=\sup \{t \in[0,1]: h(r) \geq g(s, y)-\ell \varepsilon \forall r \in[0, t]\} .
$$

By continuity of $g_{s}$, for any $t \in[0, \bar{t}]$ and $z_{t}:=y+t(x-y)$ we have

$$
h(t)=g\left(s, z_{t}\right) \geq g(s, y)-\ell \varepsilon \geq f(y)-\gamma-\ell \varepsilon \geq f(\bar{x})-\eta,
$$

hence $s \in T\left(z_{t}, \eta\right)$. In particular, taking $t=0$, we see that $s \in T(y, \eta)$, hence, by $\left(C_{3}\right),\left\|g_{s}^{\prime}\left(z_{t}\right)-g_{s}^{\prime}(y)\right\| \leq \sigma\|z-y\|^{p-1}$. It follows that $\left\|g_{s}^{\prime}\left(z_{t}\right)\right\| \leq \ell$. The mean value theorem yields some $z \in[y, y+\bar{t}(x-y)]$ such that

$$
h(0)-h(\bar{t})=\bar{t} g_{s}^{\prime}(z)(x-y) .
$$

Thus $h(0)-h(\bar{t}) \leq 4 \bar{t} \ell \delta<\ell \varepsilon$. If $\bar{t}<1$, then by continuity, we have $h(\bar{t})=$ $g(s, y)-\ell \varepsilon=h(0)-\ell \varepsilon$, a contradiction. Therefore $\bar{t}=1$ and (12) yields

$$
g(s, y)-g(s, x)=g_{s}^{\prime}(z)(x-y) \leq \ell\|x-y\| .
$$


Since $s$ is arbitrary in $S(y, \gamma)$, we get

$$
f(y)-f(x) \leq \sup _{s \in S(y, \gamma)} g(s, y)-g(s, x) \leq \ell\|x-y\|
$$

and $f$ is Lipschitzian with constant $\ell$ on $B(\bar{x}, 2 \delta)$.

Now let us estimate $f^{C}(x, u)$ for $(x, u) \in B(\bar{x}, \delta) \times S_{X}$. Since $f$ is Lipschitzian, we can pick a sequence $\left(\left(x_{n}, t_{n}\right)\right) \rightarrow\left(x, 0_{+}\right)$such that $f^{C}(x, u)=$ $\lim _{n} t_{n}^{-1}\left(f\left(x_{n}+t_{n} u\right)-f\left(x_{n}\right)\right)$. Set $y_{n}:=x_{n}+t_{n} u, \gamma_{n}:=\min \left(t_{n}^{2}, \gamma_{y_{n}}, \varepsilon\right)$ and choose $s_{n} \in S\left(x_{n}+t_{n} u, \gamma_{n}\right)$. Taking $n \geq k$, with $k$ large enough, we may suppose that $x_{n} \in B(\bar{x}, \delta)$ and $x_{n}+t_{n} u \in B(\bar{x}, \delta)$. Using relation (13), we obtain

$$
g_{s_{n}}(x) \geq g_{s_{n}}\left(y_{n}\right)-\ell\left\|x-y_{n}\right\| \geq f\left(y_{n}\right)-\gamma_{n}-\ell t_{n},
$$

hence, as $f$ is l.s.c. at $x, \liminf _{n} g_{s_{n}}(x) \geq f(x)$ or $\left(g_{s_{n}}(x)\right) \rightarrow f(x)$.

Moreover, using again (13), for every $w \in B(\bar{x}, \delta)$ and $n \geq k$ we have

$$
\begin{aligned}
g_{s_{n}}(w) & \geq g_{s_{n}}\left(y_{n}\right)-\ell\left\|w-y_{n}\right\| \geq f\left(y_{n}\right)-\gamma_{n}-2 \ell \delta \\
& \geq f(\bar{x})-2 \varepsilon-\ell \varepsilon \geq f(\bar{x})-\eta,
\end{aligned}
$$

so that $s_{n} \in T(w, \eta)$.

Taking $t \in] 0, \delta]$, from the mean value theorem we get some $r_{n}, r_{n}^{\prime} \in[0,1]$ such that

$$
\begin{aligned}
\frac{1}{t}\left(g\left(s_{n}, x+t u\right)-g\left(s_{n}, x\right)\right) & =g_{s_{n}}^{\prime}\left(x+r_{n} t u\right)(u), \\
\frac{1}{t_{n}}\left(g\left(s_{n}, x_{n}+t_{n} u\right)-g\left(s_{n}, x_{n}\right)\right) & =g_{s_{n}}^{\prime}\left(x_{n}+r_{n}^{\prime} t_{n} u\right)(u) .
\end{aligned}
$$

Choosing $w=x+r_{n} t u$ and then $w=x_{n}+r_{n}^{\prime} t_{n} u$ in (14), we find that $s_{n} \in T\left(x+r_{n} t u, \eta\right) \cap T\left(x_{n}+r_{n}^{\prime} t_{n} u, \eta\right)$, so that we may apply $\left(C_{3}\right)$. Thus

$$
\begin{aligned}
& \frac{1}{t_{n}}\left(g\left(s_{n}, x_{n}+t_{n} u\right)-g\left(s_{n}, x_{n}\right)\right)-\frac{1}{t}\left(g\left(s_{n}, x+t u\right)-g\left(s_{n}, x\right)\right) \\
& \quad \leq\left\|g_{s_{n}}^{\prime}\left(x_{n}+r_{n}^{\prime} t_{n} u\right)-g_{s_{n}}^{\prime}\left(x+r_{n} t u\right)\right\| \leq \sigma\left\|x_{n}-x+r_{n}^{\prime} t_{n} u-r_{n} t u\right\|^{p-1} .
\end{aligned}
$$

From these inequalities, the choice of $s_{n}$ and the fact that $\left(g\left(s_{n}, x\right)\right) \rightarrow f(x)$, we deduce that

$$
\begin{aligned}
\lim _{n} \frac{1}{t_{n}} & \left(f\left(x_{n}+t_{n} u\right)-f\left(x_{n}\right)\right) \leq \liminf _{n} \frac{1}{t_{n}}\left(g\left(s_{n}, x_{n}+t_{n} u\right)-g\left(s_{n}, x_{n}\right)+t_{n}^{2}\right) \\
& \leq \liminf _{n}\left[\frac{1}{t}\left(g\left(s_{n}, x+t u\right)-g\left(s_{n}, x\right)\right)+\sigma\left\|x_{n}-x+r_{n}^{\prime} t_{n} u-r_{n} t u\right\|^{p-1}\right] \\
& \leq \frac{1}{t}(f(x+t u)-f(x))+\sigma t^{p-1}\|u\|^{p-1} .
\end{aligned}
$$

Therefore, for any $v \in \delta \bar{B}_{X}$, setting $v=t u$ with $\left.\left.t \in\right] 0, \delta\right]$ and $u \in S_{X}$, we get (11). 
The following immediate consequence partially generalizes [14, Thm. 5.1] which is set in finite-dimensional spaces and for suprema of functions of class $C^{2}$.

Corollary 12. Suppose $S$ is a compact topological space, and $g: S \times X$ $\rightarrow \mathbb{R}$ is continuous with a partial derivative with respect to $x \in B(\bar{x}, \varrho)$ which is jointly continuous and Lipschitz with respect to $x$, uniformly with respect to $s \in S$. Then the marginal function $f$ is Lipschitzian and 2-paraconvex on $B(\bar{x}, \delta)$ for some $\delta>0$.

Another consequence will be used later on.

Corollary 13. Suppose $X$ is a uniformly smooth Banach space with modulus of smoothness $\varrho_{X}$ satisfying for some $k>0$ and $p>1$ the estimate $\varrho_{X}(t) \leq k t^{p}$ for $t \geq 0$. Then, for any nonempty closed subset $E$ of $X$, the function $-d_{E}$ is p-paraconvex on $X \backslash E$.

Proof. Let $j$ be the derivative of the norm on $X \backslash\{0\}$. Let us first prove that, given $s>r>0$, there exists some $c>0$ such that

$$
\|j(x)-j(y)\| \leq c\|x-y\|^{p-1} \quad \forall x, y \in s \bar{B}_{X} \backslash r \bar{B}_{X} .
$$

By [6, Prop. A.5] we have, for any $x, y \in s \bar{B}_{X} \backslash r \bar{B}_{X}$,

$$
\begin{aligned}
\|j(x)-j(y)\| & \leq k 2^{p}\left\|\frac{x}{\|x\|}-\frac{y}{\|y\|}\right\|^{p-1} \leq k 2^{p} r^{2-2 p}\|\| y\|x-\| x\|y\|^{p-1} \\
& \leq k 2^{p} r^{2-2 p}(\|y\|\|x-y\|+\|y\||\|x\|-\|y\||)^{p-1} \\
& \leq k 2^{2 p-1} r^{2-2 p} s^{p-1}\|x-y\|^{p-1} .
\end{aligned}
$$

Given $\bar{x} \in X \backslash E$, let $\eta=\varrho=r=d_{E}(\bar{x}) / 2$ and set $S:=E, g(e, x)=-\|x-e\|$ for $x \in B(\bar{x}, \varrho)$ and $e \in E$. Then $f(x)=-d_{E}(x)$ for $x \in B(\bar{x}, \varrho)$, and for $e \in T(x, \eta)$, i.e. for $e \in E$ satisfying $\|x-e\| \leq d_{E}(\bar{x})+\eta$, we have $r \leq\|x-e\| \leq 3 r$. Thus (15) shows that condition $\left(C_{3}\right)$ is satisfied. Since the norm is differentiable on $B(\bar{x}, \varrho)$ with derivative $j$ bounded by 1 , conditions $\left(C_{1}\right)$ and $\left(C_{2}\right)$ are also satisfied.

5. Paraconvexity of sets. We observe that using the notion of $p$ paraconvexity for the indicator function $\iota_{E}$ of a subset $E$ of $X$ would lead to convexity of $E$ and not to a relaxed form of convexity. Therefore, we rather use the distance function $d_{E}$. In the following, $\bar{x}$ is a point of $E$ and $p \in] 1,2]$.

Definition 14. A subset $E$ of $X$ is said to be p-paraconvex around $\bar{x}$ if its associated distance function $d_{E}$ is $p$-paraconvex around $\bar{x}$.

This notion is different from the one studied in [35]. Clearly, a $p$-paraconvex set is approximately convex in the sense of [38]. 
ExAmple. The set $E:=\left\{(r, s) \in \mathbb{R}^{2}: s \geq|r|-r^{2}\right\}$ is $p$-paraconvex but nonconvex. This example is a special instance of Proposition 25 below.

It is not obvious to decide whether the preceding definition depends on the choice of the norm in an equivalence class; by contrast, the variant presented in the next section will not depend on the choice of the norm inducing the topology.

The following result is an easy consequence of [18] when $\partial=\partial^{C}$. However, since we use here an arbitrary subdifferential contained in the Clarke subdifferential, we have to use Theorem 8 with the distance function.

THEOREM 15. Let $\partial$ be a subdifferential on the family of Lipschitz functions such that $\partial f \subset \partial^{C} f$ for any Lipschitz function $f$ on $X$. Then $(\mathrm{a}) \Rightarrow(\mathrm{b}) \Rightarrow(\mathrm{c}) \Leftrightarrow\left(\mathrm{c}^{\prime}\right) \Rightarrow(\mathrm{d})$ below. If moreover $\partial$ is Lipschitz-valuable on $X$, all these assertions are equivalent.

(a) $E$ is p-paraconvex around $\bar{x}$ in the sense that $d_{E}$ is p-paraconvex around $\bar{x}$;

(b) there exist $c, \varrho>0$ such that for any $x \in B(\bar{x}, \varrho)$ and $v \in B(0, \varrho)$,

$$
d_{E}^{C}(x, v) \leq d_{E}(x+v)-d_{E}(x)+c\|v\|^{p} ;
$$

(c) there exist $c, \varrho>0$ such that for any $x \in B(\bar{x}, \varrho), x^{*} \in \partial d_{E}(x)$ and $\left.\left.(u, t) \in S_{X} \times\right] 0, \varrho\right)$

$$
\left\langle x^{*}, u\right\rangle \leq \frac{d_{E}(x+t u)-d_{E}(x)}{t}+c t^{p-1}
$$

$\left(\mathrm{c}^{\prime}\right)$ there exist $c, \varrho>0$ such that for any $x \in B(\bar{x}, \varrho), x^{*} \in \partial d_{E}(x)$ and $v \in \varrho \bar{B}_{X}$

$$
\left\langle x^{*}, v\right\rangle \leq d_{E}(x+v)-d_{E}(x)+c\|v\|^{p}
$$

(d) $\partial d_{E}$ is p-paramonotone around $\bar{x}$.

The following corollary is a consequence of Corollary 9.

Corollary 16. If $E$ is p-paraconvex around $\bar{x}$ for some $p>1$, then, for any subdifferential $\partial$ such that $\partial^{p} f \subset \partial f \subset \partial^{C} f$ for any Lipschitz function $f$, one has $\partial^{p} d_{E}(x)=\partial^{F} d_{E}(x)=\partial d_{E}(x)=\partial^{C} d_{E}(x)$ for $x$ close to $\bar{x}$.

6. Intrinsic $p$-paraconvexity. As in the case of approximate convexity $([40])$, the terminology of the definition we now adopt is justified by the fact that the notion we introduce is obtained by restricting the requirement on the distance function to the subset $E$. Thus, this new notion is more general than the preceding one. We will show later that the two notions do not coincide.

Definition 17. Given $p \in[1,+\infty)$, a subset $E$ of $X$ is said to be $i n$ trinsically $p$-paraconvex around $\bar{x} \in E$ if there exist $c, \varrho>0$ such that for 
any $x_{1}, x_{2} \in E \cap B(\bar{x}, \varrho)$ and $t \in[0,1]$,

$$
d_{E}\left((1-t) x_{1}+t x_{2}\right) \leq c t(1-t)\left\|x_{1}-x_{2}\right\|^{p} .
$$

It is intrinsically p-paraconvex if it is intrinsically $p$-paraconvex around each of its points.

Note that there is no loss of generality in assuming that $E$ is closed. Also note that this definition does not depend on the choice of the norm among the ones inducing the same topology.

Characterizations can be given as follows. When one of the assertions (c)-(d) below holds, we say that $E$ is $\partial$-intrinsically p-paraconvex around $\bar{x}$.

THEOREM 18. Let $E$ be a nonempty closed subset of $X$ and let $\partial$ be a subdifferential such that $\partial f \subset \partial^{C} f$ for any Lipschitz function $f$ on $X$. Then $(\mathrm{a}) \Rightarrow(\mathrm{b}) \Rightarrow(\mathrm{c}) \Leftrightarrow(\mathrm{d}) \Leftarrow(\mathrm{e})$, and when $X$ is a $\partial$-subdifferentiability space, also $(\mathrm{e}) \Rightarrow(\mathrm{a})$, where

(a) $E$ is intrinsically $p$-paraconvex around $\bar{x}$;

(b) there exist $c, \delta>0$ such that for any $x, x^{\prime} \in E \cap B(\bar{x}, \delta)$,

$$
d_{E}^{C}\left(x, x^{\prime}-x\right) \leq c\left\|x-x^{\prime}\right\|^{p}
$$

(c) there exist $c, \delta>0$ such that for any $x, x^{\prime} \in E \cap B(\bar{x}, \delta)$ and $x^{*} \in$ $\partial d_{E}(x)$

(d) $\partial d_{E}(\cdot)$ is p-paramonotone around $\bar{x}$ on $E$ : there exist $c, \delta>0$ such that for any $x_{1}, x_{2} \in E \cap B(\bar{x}, \delta), x_{1}^{*} \in \partial d_{E}\left(x_{1}\right)$ and $x_{2}^{*} \in \partial d_{E}\left(x_{2}\right)$,

$$
\left\langle x_{1}^{*}-x_{2}^{*}, x_{1}-x_{2}\right\rangle \geq-c\left\|x_{1}-x_{2}\right\|^{p} ;
$$

(e) there exist $c, \varrho>0$ such that for any $w \in B(\bar{x}, \varrho), x \in E \cap B(\bar{x}, \varrho)$ and $w^{*} \in \partial d_{E}(w)$,

$$
d_{E}(w)+\left\langle w^{*}, x-w\right\rangle \leq c\|x-w\|^{p} .
$$

Proof. $(\mathrm{a}) \Rightarrow(\mathrm{b})$. Let $\varrho>0$ be as in Definition 17 and let $x, x^{\prime} \in E \cap$ $B(\bar{x}, \varrho)$. By Lemma 5 , we have

$$
d_{E}^{C}\left(x, x^{\prime}-x\right) \leq \limsup _{t \rightarrow 0_{+}, e \stackrel{E}{\rightarrow} x} \frac{1}{t} d_{E}\left(e+t\left(x^{\prime}-x\right)\right) .
$$

Now, since for $t \in] 0,1\left[, d_{E}\left(e+t\left(x^{\prime}-x\right)\right) \leq d_{E}\left(e+t\left(x^{\prime}-e\right)\right)+t\|e-x\| \leq\right.$ $c t(1-t)\left\|x^{\prime}-e\right\|^{p}+t\|e-x\|$, we get

$$
d_{E}^{C}\left(x, x^{\prime}-x\right) \leq c\left\|x^{\prime}-x\right\|^{p} .
$$

(b) $\Rightarrow(\mathrm{c})$ is a consequence of the inclusion $\partial d_{E}(x) \subset \partial^{C} d_{E}(x)$ and of the definition of $\partial^{C} d_{E}(x)$. When $\partial=\partial^{C}$, the reverse implication follows from the relation $d_{E}^{C}(x, u)=\sup \left\{\left\langle x^{*}, u\right\rangle: x^{*} \in \partial^{C} d_{E}(x)\right\}$. 
$(\mathrm{c}) \Rightarrow(\mathrm{d})$. Let $c, \delta>0$ be as in (c) and let $x_{1}, x_{2} \in E \cap B(\bar{x}, \delta), x_{1}^{*} \in$ $\partial d_{E}\left(x_{1}\right)$ and $x_{2}^{*} \in \partial d_{E}\left(x_{2}\right)$. Taking $x=x_{1}, x^{*}=x_{1}^{*}, x^{\prime}=x_{2}$ and adding inequality (21) to the corresponding one obtained by choosing $x=x_{2}$, $x^{*}=x_{2}^{*}, x^{\prime}=x_{1}$ we get $(22)$ with $2 c$ in place of $c$.

$(\mathrm{d}) \Rightarrow(\mathrm{c})$ is obtained by taking $x_{1}=x, x_{1}^{*}=x^{*}, x_{2}=x^{\prime}, x_{2}^{*}=0$ in (d), using the fact that $x_{2}$ is a minimizer of $d_{E}$, so that $0 \in \partial d_{E}\left(x_{2}\right)$.

(e) $\Rightarrow(\mathrm{c})$ is obvious (take $\left.w=x^{\prime} \in E\right)$.

(e) $\Rightarrow$ (a) when $X$ is a $\partial$-subdifferentiability space. Given $c, \varrho>0$ as in (e), let $x_{1}, x_{2} \in E \cap B(\bar{x}, \varrho), t \in[0,1]$, and $w:=(1-t) x_{1}+t x_{2}$. Since $X$ is a $\partial$-subdifferentiability space, there exist sequences $\left(w_{n}\right) \rightarrow w$ and $\left(w_{n}^{*}\right)$ such that $w_{n}^{*} \in \partial d_{E}\left(w_{n}\right)$ for each $n \in \mathbb{N}$. Then, as $\partial d_{E}\left(w_{n}\right) \subset \partial^{C} d_{E}\left(w_{n}\right) \subset \bar{B}_{X^{*}}$, we have $\left(\left\langle w_{n}^{*}, w-w_{n}\right\rangle\right) \rightarrow 0$. Since by convexity $w \in B(\bar{x}, \varrho)$, we have $w_{n} \in B(\bar{x}, \varrho)$ for $n$ large enough, it follows that

$$
\begin{aligned}
(1-t) d_{E}\left(w_{n}\right)+(1-t)\left\langle w_{n}^{*}, x_{1}-w_{n}\right\rangle & \leq(1-t) c\left\|x_{1}-w_{n}\right\|^{p} \\
t d_{E}\left(w_{n}\right)+t\left\langle w_{n}^{*}, x_{2}-w_{n}\right\rangle & \leq t c\left\|x_{2}-w_{n}\right\|^{p} .
\end{aligned}
$$

Adding these relations, we get

$$
d_{E}\left(w_{n}\right)+\left\langle w_{n}^{*}, w-w_{n}\right\rangle \leq(1-t) c\left\|x_{1}-w_{n}\right\|^{p}+t c\left\|x_{2}-w_{n}\right\|^{p},
$$

and, passing to the limit,

$d_{E}(w) \leq c(1-t) t^{p}\left\|x_{1}-x_{2}\right\|^{p}+c t(1-t)^{p}\left\|x_{2}-x_{1}\right\|^{p} \leq 2 c t(1-t)\left\|x_{1}-x_{2}\right\|^{p}$.

Now let us give specializations to some specific subdifferentials and normal cones. We start with the Fréchet normal cone.

Conollary 19. For the Fréchet subdifferential $\partial^{F}$, assertion (c) of the preceding theorem is equivalent to the following:

(f) there exist $c, \delta>0$ such that for any $x, x^{\prime} \in E \cap B(\bar{x}, \delta)$ and $x^{*} \in$ $N^{F}(E, x)$,

$$
\left\langle x^{*}, x^{\prime}-x\right\rangle \leq c\left\|x^{*}\right\|\left\|x-x^{\prime}\right\|^{p} .
$$

If (c) holds with $\partial=\partial^{C}$, then $N^{C}(E, x)=N^{F}(E, x)$ for all $x \in E \cap B(\bar{x}, \delta)$.

If $X$ is an Asplund space then all the assertions of Theorem 18 are equivalent to (f).

Proof. The relation $\partial^{F} d_{E}(x)=N^{F}(E, x) \cap \bar{B}_{X^{*}}$ for each $x \in E$ yields equivalence of (c) and (f) by a homogeneity argument which does not require any assumption on $X$. Moreover, (c) shows that $\partial^{C} d_{E}(x)=\partial^{F} d_{E}(x)$ for each $x \in E \cap B(\bar{x}, \delta)$ and that $\partial^{F} d_{E}(x)$ is weak* closed. Therefore, by [13, Prop. 2.4.2], denoting by $\overline{\mathrm{co}}^{*}(S)$ the weak* closed convex hull of a subset $S$ of $X^{*}$, we get

$$
N^{C}(E, x)=\overline{\mathrm{co}}^{*}\left(\mathbb{R}_{+} \partial^{C} d_{E}(x)\right)=\overline{\mathrm{co}}^{*}\left(\mathbb{R}_{+} \partial^{F} d_{E}(x)\right)=N^{F}(E, x)
$$

since $N^{F}(E, x)$ is convex and weak* closed by (f). 
(c) $\Rightarrow(\mathrm{e})$ when $X$ is an Asplund space. Let $c, \delta>0$ be as in (c). Let $\varrho:=\delta / 2, w \in B(\bar{x}, \varrho) \backslash E$ (note that $(23)$ coincides with $(21)$ if $w \in E$ ), $x \in E \cap B(\bar{x}, \varrho)$ and $w^{*} \in \partial^{F} d_{E}(w)$. By Lemma 6 we can find sequences $\left(x_{n}\right)$ in $E$ and $\left(x_{n}^{*}\right)$ in $X^{*}$ such that $x_{n}^{*} \in \partial^{F} d_{E}\left(x_{n}\right)$ for each $n \in \mathbb{N}$ and

$$
\left(\left\|x_{n}-w\right\|\right) \rightarrow d_{E}(w), \quad\left(\left\langle x_{n}^{*}, w-x_{n}\right\rangle\right) \rightarrow d_{E}(w), \quad\left(\left\|x_{n}^{*}-w^{*}\right\|\right) \rightarrow 0 .
$$

Since $d_{E}(w) \leq\|\bar{x}-w\|<\varrho$ and $\|x-w\|>0$, we may suppose that $\left\|x_{n}-w\right\| \leq$ $\varrho,\left\|x_{n}-w\right\| \leq 2\|x-w\|$ for each $n \in \mathbb{N}$; then $x_{n} \in B(\bar{x}, 2 \varrho) \subset B(\bar{x}, \delta)$ and $\left\|x-x_{n}\right\| \leq\|x-w\|+\left\|w-x_{n}\right\| \leq 3\|x-w\|$. Now, for $n$ large enough, (25) implies the first inequality below, while (c) ensures the third:

$$
\begin{aligned}
d_{E}(w) & +\left\langle w^{*}, x-w\right\rangle \\
& \leq \underset{n}{\lim \sup }\left(\left\langle x_{n}^{*}, w-x_{n}\right\rangle+\left(\left\langle x_{n}^{*}, x-w\right\rangle+\left\|x_{n}^{*}-w^{*}\right\|\|x-w\|\right)\right) \\
& \leq \underset{n}{\lim \sup }\left(\left\langle x_{n}^{*}, x-x_{n}\right\rangle+\left\|x_{n}^{*}-w^{*}\right\|\|x-w\|\right) \\
& \leq \limsup _{n}\left(c\left\|x-x_{n}\right\|^{p}+\left\|x_{n}^{*}-w^{*}\right\|\|x-w\|\right) .
\end{aligned}
$$

Passing to the limit, and using the estimate $\left\|x-x_{n}\right\| \leq 3\|x-w\|$ obtained above, we get

$$
d_{E}(w)+\left\langle w^{*}, x-w\right\rangle \leq c\left(3^{p}+1\right)\|x-w\|^{p} .
$$

Finally, since $X$ is Asplund, it is a $\partial^{F}$-subdifferentiability space, so that (e) ensures that $E$ is intrinsically $p$-paraconvex by Theorem 18 .

The case of the limiting subdifferential can easily be derived from the preceding corollary.

Corollary 20. Suppose that $E$ is a closed subset of an Asplund space $X$ and let $\partial$ be the limiting Fréchet subdifferential $\overline{\partial^{F}}$. Then all the assertions of Theorem 18 are equivalent.

Proof. In view of Corollary 7 , the passages from the assertions using $\partial^{F}$ to those involving $\overline{\partial^{F}}$ result from a passage to the weak* limit for a bounded net; the reverse implications are obvious.

Now let us turn to the Clarke subdifferential. It would be interesting to know whether one can get rid of the assumption of the last assertion of the next corollary that $X$ is an Asplund space.

Corollary 21. For a closed subset $E$ of a Banach space $X$ and $\partial=\partial^{C}$, we have $(\mathrm{e}) \Rightarrow(\mathrm{a}) \Rightarrow(\mathrm{b}) \Leftrightarrow(\mathrm{c}) \Leftrightarrow(\mathrm{d})$ in Theorem 18. If moreover $X$ is an Asplund space, all these assertions are equivalent.

Proof. The implications follow from the choice $\partial=\partial^{C}$ in Theorem 18 since any Banach space is a $\partial^{C}$-subdifferentiability space. When $X$ is an Asplund space the equivalences can be deduced from the preceding corollary: 
since $\partial^{C} d_{E}(x)=\overline{\operatorname{co}}^{*}\left(\overline{\partial^{F}} d_{E}(x)\right)$ one has the equivalence $\left(\mathrm{e}^{F}\right) \Leftrightarrow\left(\overline{\mathrm{e}^{F}}\right) \Leftrightarrow\left(\mathrm{e}^{C}\right)$ (where $\left(\mathrm{e}^{C}\right),\left(\overline{\mathrm{e}^{F}}\right),\left(\mathrm{e}^{F}\right)$ are (e) for $\partial=\partial^{C}, \overline{\partial^{F}}, \partial^{F}$ respectively), hence $\left(\mathrm{c}^{C}\right) \Rightarrow\left(\mathrm{c}^{F}\right) \Rightarrow\left(\mathrm{e}^{F}\right) \Rightarrow\left(\mathrm{e}^{C}\right) \Rightarrow(\mathrm{a})$.

The preceding local property can be related to a global one as defined and studied in [10], [15], [19], [20], [22] in the case $p=2, X$ being a Hilbert space.

Definition 22. Given a subset $E$ of $X, p \in] 1,2]$ and a continuous function $\varphi: E \times E \rightarrow \mathbb{R}_{+}$, the subset $E$ of $X$ is said to be $\varphi$-p-convex if for any $x, y \in E$ and $x^{*} \in N^{F}(E, x)$,

$$
\left\langle x^{*}, y-x\right\rangle \leq \varphi(x, y)\left\|x^{*}\right\|\|x-y\|^{p} .
$$

In this definition the choice of the Fréchet normal cone is natural since if the above condition holds for some other cone $N(E, x)$ contained in $N^{C}(E, x)$ then $N(E, x) \subset N^{F}(E, x)$. The following result clarifies the links between $\varphi$-p-convexity and $p$-paraconvexity.

Proposition 23. Let $p \in] 1,2], E$ a subset of a Banach space $X$, and $\varphi: E \times E \rightarrow \mathbb{R}_{+}$a continuous function such that $E$ is $\varphi$-p-convex. Then $E$ is $\partial^{F}$-intrinsically p-paraconvex around each point of $E$ in the sense that assertion (c) of Theorem 18 is satisfied with $\partial=\partial^{F}$.

Conversely, if $E$ is $\partial^{F}$-intrinsically p-paraconvex around each point of $E$, then $E$ is $\varphi$-p-convex for some continuous function $\varphi: E \times E \rightarrow \mathbb{R}_{+}$.

Proof. The first assertion is immediate: given $c>\varphi(\bar{x}, \bar{x})$ for some $\bar{x} \in E$, relation (21) is satisfied whenever $\delta>0$ is small enough. Let us prove the converse by first showing that there exist an open neighborhood $V$ of the diagonal of $E \times E$ and a continuous function $\psi: V \rightarrow \mathbb{R}_{+}$such that for any $(x, y) \in V$ and $x^{*} \in N^{F}(E, x)$,

$$
\left\langle x^{*}, y-x\right\rangle \leq \psi(x, y)\left\|x^{*}\right\|\|x-y\|^{p} .
$$

Given $(x, y) \in E \times E$, let

$$
\omega_{p}(x, y)=\sup \left\{\left\langle x^{*}, \frac{y-x}{\|x-y\|^{p}}\right\rangle: x^{*} \in N^{F}(E, x) \cap \bar{B}_{X^{*}}\right\} .
$$

Assertion (c) of Theorem 18 shows that for any $e \in E$ there exist $c, \varrho>0$ such that $\omega_{p}(x, y) \leq c$ for any $x, y \in B(e, \varrho)$. Clearly, the infimum $c_{p}(e)$ of such constants $c$ defines an upper semicontinuous function on $E$. Since $E$ is a metric space, there exists a continuous function $\psi_{p}: E \rightarrow \mathbb{R}$ such that $c_{p}(e)+1 \leq \psi_{p}(e)$ for every $e \in E$. Then, by definition of $c_{p}$, for each $e \in E$ one can find $\varrho(e)>0$ such that $\omega_{p}(x, y) \leq c_{p}(e)+1 \leq \psi_{p}(e)$ for all $x, y \in B(e, \varrho(e))$. Since $E$ is paracompact, we can find a locally finite open 
covering $\left(V_{e}\right)_{e \in E}$ of $E$ such that $V_{e} \subset B(e, \varrho(e))$ for every $e \in E$. Set

$$
V:=\bigcup_{e \in E} V_{e} \times V_{e}, \quad \psi(x, y):=\sum_{e \in E} \lambda_{e}(x, y) \psi_{p}(e),
$$

where $\left(\lambda_{e}\right)_{e \in E}$ is a continuous partition of unity subordinated to the covering $\left(V_{e} \times V_{e}\right)_{e \in E}$ of $V$. Then $\psi$ is continuous and for every $(x, y) \in V$ one has

$$
\omega_{p}(x, y)=\sum_{e \in E} \lambda_{e}(x, y) \omega_{p}(x, y) \leq \sum_{e \in E} \lambda_{e}(x, y) \psi_{p}(e)=\psi(x, y),
$$

so that inequality (27) holds.

Let $U$ be a closed neighborhood of the diagonal of $E \times E$ contained in $V$ and let $\varphi$ be given by

$$
\varphi(x, y)=(1-t(x, y)) \psi(x, y)+t(x, y) \frac{1}{\|x-y\|^{p-1}}
$$

where

$$
t(x, y):=\frac{d((x, y), U)}{d((x, y),(E \times E) \backslash V)+d((x, y), U)} .
$$

Then $\varphi$ is a continuous function on $E \times E$. For $(x, y) \in U$ and $x^{*} \in N(E, x)$,

$$
\left\langle x^{*}, \frac{y-x}{\|x-y\|^{p}}\right\rangle \leq\left\|x^{*}\right\| \psi(x, y)=\left\|x^{*}\right\| \varphi(x, y) .
$$

For $(x, y) \in(E \times E) \backslash V$ and $x^{*} \in N(E, x)$,

$$
\left\langle x^{*}, \frac{y-x}{\|x-y\|^{p}}\right\rangle \leq\left\|x^{*}\right\| \frac{1}{\|x-y\|^{p-1}}=\left\|x^{*}\right\| \varphi(x, y) .
$$

For $(x, y) \in V \backslash U, x^{*} \in N(E, x)$,

$$
\left\langle x^{*}, \frac{y-x}{\|x-y\|^{p}}\right\rangle \leq \min \left(\left\|x^{*}\right\| \frac{1}{\|x-y\|^{p-1}},\left\|x^{*}\right\| \psi(x, y)\right) \leq\left\|x^{*}\right\| \varphi(x, y) .
$$

Therefore (26) is satisfied.

The following statement can be considered as an illustration of the use of a global property as in the preceding definition.

Proposition 24. Let $p \geq q \in[1, \infty)$, let $(S, \mathcal{S}, \mu)$ be a measure space with a finite measure, let $c \in L_{\infty}(S, \mathbb{R})$ and let $F: S \rightrightarrows \mathbb{R}^{n}$ be a measurable multifunction such that

$\forall s \in S, \forall x, y \in F(s), \forall t \in[0,1]: d_{F(s)}((1-t) x+t y) \leq c(s) t(1-t)\|x-y\|^{p / q}$.

Then the set $E$ of measurable selections of $F(\cdot)$ which are in $L_{p}\left(S, \mathbb{R}^{n}\right)$ considered as a subset of $X:=L_{q}\left(S, \mathbb{R}^{n}\right)$ is such that

$$
\forall u, v \in E, \forall t \in[0,1]: d_{X}((1-t) u+t v, E) \leq\|c\|_{\infty} t(1-t)\|u-v\|_{p}^{p / q} .
$$


Proof. Let $u, v \in E$, and for $t \in[0,1]$, let $w:=(1-t) u+t v$. Then, by [55, Thm. 14.60], we can interchange minimization and integration to get

$$
\begin{aligned}
d_{X}(w, E)^{q} & :=\inf _{z \in E} \int_{S}\|w(s)-z(s)\|^{q} \mu(d s)=\int_{S} \inf _{e \in F(s)}\|w(s)-e\|^{q} \mu(d s) \\
& \leq \int_{S} c(s)^{q} t^{q}(1-t)^{q}\|u(s)-v(s)\|^{p} \mu(d s) \\
& \leq\|c\|_{\infty}^{q} t^{q}(1-t)^{q}\|u-v\|_{p}^{p} .
\end{aligned}
$$

Inequality (28) follows.

7. Links between $p$-paraconvex sets and functions. Some links between geometrical properties and analytical properties are contained in the next statements. In the present section we suppose $p>1$. Unless otherwise specified, we endow the product space $X:=W \times \mathbb{R}$ of a n.v.s. $W$ with $\mathbb{R}$ with a product norm, i.e. a norm such that the projections and the injections $w \mapsto(w, 0)$ and $r \mapsto(0, r)$ are nonexpansive. Then for each $(w, r) \in W \times \mathbb{R}$ we have

$$
\max (\|w\|,|r|) \leq\|(w, r)\| \leq\|w\|+|r| .
$$

Proposition 25. Let $W$ be a normed vector space and let $f: W \rightarrow \mathbb{R}_{\infty}$ be a l.s.c. function which is p-paraconvex around $\bar{w} \in W$. Then for any $\bar{r} \geq$ $f(\bar{w})$ the epigraph $E$ of $f$ is intrinsically p-paraconvex around $\bar{x}:=(\bar{w}, \bar{r})$.

Proof. Let $c, \varrho>0$ be such that

$$
f\left((1-t) w_{1}+t w_{2}\right) \leq(1-t) f\left(w_{1}\right)+t f\left(w_{2}\right)+c t(1-t)\left\|w_{1}-w_{2}\right\|^{p}
$$

for any $w_{1}, w_{2} \in B(\bar{w}, \varrho), t \in[0,1]$. Let $x_{i}:=\left(w_{i}, r_{i}\right)(i=1,2)$ be elements of the epigraph $E$ of $f$ in $B(\bar{x}, \varrho)$ and let $t \in[0,1], w:=(1-t) w_{1}+t w_{2}, r:=$ $(1-t) r_{1}+t r_{2}$ and $x:=(w, r)$. Then, as $w_{1}, w_{2} \in B(\bar{w}, \varrho)$ and $(w, f(w)) \in E$, one has $d_{E}(x)=0$ if $f(w) \leq r$ and $d_{E}(x) \leq\|(w, r)-(w, f(w))\| \leq f(w)-r$ if $f(w)>r$, so that

$$
\begin{aligned}
d_{E}(x) & \leq \max (0, f(w)-r) \\
& \leq \max \left(0,(1-t)\left(f\left(w_{1}\right)-r_{1}\right)+t\left(f\left(w_{2}\right)-r_{2}\right)+c t(1-t)\left\|w_{1}-w_{2}\right\|^{p}\right) \\
& \leq c t(1-t)\left\|w_{1}-w_{2}\right\|^{p} \leq c t(1-t)\left\|x_{1}-x_{2}\right\|^{p} .
\end{aligned}
$$

Thus $E$ is intrinsically $p$-paraconvex around $\bar{x}$.

Let us complete the preceding result with the following one.

Proposition 26. Let $f: W \rightarrow \mathbb{R}$ be a function which is Lipschitzian with constant $\ell>0$ on some ball $B(\bar{w}, \varrho)$. Suppose $X:=W \times \mathbb{R}$ is endowed with the norm given by $\|(w, r)\|=\ell\|w\|+|r|$. If $f$ is $p$-paraconvex around $\bar{w}$, then, for any $\bar{r} \geq f(\bar{w})$, the epigraph $E$ of $f$ is p-paraconvex around $\bar{x}:=$ $(\bar{w}, \bar{r})$. 
Proof. We may suppose $\bar{r}=f(\bar{w})$ since otherwise $\bar{x}$ is in the interior of $E$. By [23], [26] one can find $\left.\varrho^{\prime} \in\right] 0, \varrho[$ such that

$$
d_{E}(w, r)=(f(w)-r)_{+}
$$

for $(w, r) \in B\left(\bar{x}, \varrho^{\prime}\right)$ with $\bar{x}:=(\bar{w}, f(\bar{w})), X$ being endowed with the norm described in the statement; here, for $t \in \mathbb{R}, t_{+}$stands for $\max (t, 0)$. Let $c>0$ and $\delta \in] 0, \varrho^{\prime}[$ be such that

$$
f\left((1-t) w_{1}+t w_{2}\right) \leq(1-t) f\left(w_{1}\right)+t f\left(w_{2}\right)+c t(1-t)\left\|w_{1}-w_{2}\right\|^{p}
$$

for any $w_{1}, w_{2} \in B(\bar{w}, \delta), t \in[0,1]$. Let $x_{i}:=\left(w_{i}, r_{i}\right) \in B(\bar{x}, \delta)$ for $i=1,2$ and let $w:=(1-t) w_{1}+t w_{2}, r:=(1-t) r_{1}+t r_{2}$ and $x:=(w, r)$. Then we have $w_{1}, w_{2} \in B(\bar{w}, \delta)$ and

$$
f(w)-r \leq(1-t)\left(f\left(w_{1}\right)-r_{1}\right)_{+}+t\left(f\left(w_{2}\right)-r_{2}\right)_{+}+c t(1-t)\left\|w_{1}-w_{2}\right\|^{p},
$$

hence

$$
d_{E}(x) \leq(1-t) d_{E}\left(x_{1}\right)+t d_{E}\left(x_{2}\right)+c \ell^{-p} t(1-t)\left\|x_{1}-x_{2}\right\|^{p}
$$

Let us give a kind of converse to the preceding propositions.

THEOREM 27. Let $W$ be a Banach space and let $f: W \rightarrow \mathbb{R}$ be a function which is locally Lipschitzian around $\bar{w} \in W$ and such that the epigraph $E$ of $f$ is an intrinsically p-paraconvex subset of $X:=W \times \mathbb{R}$ around $\bar{x}:=(\bar{w}, f(\bar{w}))$. Then $f$ is p-paraconvex around $\bar{w}$.

Proof in the case $W$ is an Asplund space. In view of the characterization of $p$-paraconvexity of a function given in Theorem 8 , it suffices to prove that $\partial^{F} f$ is $p$-paramonotone around $\bar{w}$. Let $\ell$ be the Lipschitz constant of $f$ on some ball $B\left(\bar{x}, \varrho_{0}\right)$. By Corollary 19 there exist $c>0$ and $\left.\varrho \in\right] 0, \varrho_{0}[$ such that for any $x_{1}, x_{2} \in E \cap B(\bar{x}, \varrho)$ and any $x_{1}^{*} \in N^{F}\left(E, x_{1}\right), x_{2}^{*} \in N^{F}\left(E, x_{2}\right)$,

$$
\left\langle x_{1}^{*}-x_{2}^{*}, x_{1}-x_{2}\right\rangle \geq-c\left\|x_{1}-x_{2}\right\|^{p} .
$$

Let $\varrho^{\prime}:=\varrho /(\ell+1)$. Then, for $w_{1}, w_{2} \in B\left(\bar{w}, \varrho^{\prime}\right), w_{i}^{*} \in \partial^{F} f\left(w_{i}\right)$ for $i=1,2$, setting $x_{i}:=\left(w_{i}, f\left(w_{i}\right)\right), x_{i}^{*}:=\left(w_{i}^{*},-1\right)$, one has $x_{i} \in B(\bar{x}, \varrho)$ and $x_{i}^{*} \in$ $N^{F}\left(E, x_{i}\right)$, hence

$$
\begin{aligned}
\left\langle w_{1}^{*}-w_{2}^{*}, w_{1}-w_{2}\right\rangle & =\left\langle x_{1}^{*}-x_{2}^{*}, x_{1}-x_{2}\right\rangle \geq-c\left\|x_{1}-x_{2}\right\|^{p} \\
& \geq-c(\ell+1)^{p}\left\|w_{1}-w_{2}\right\|^{p} .
\end{aligned}
$$

Thus $f$ is $p$-paraconvex around $\bar{w}$.

The proof in the general case relies on the following lemma extracted from the proof of [48, Prop. $10 \mathrm{c}]$; it is close to previous results of that kind due to F. H. Clarke [13] and to A. D. Ioffe [27] in the case $\bar{x}=(\bar{w}, f(\bar{w}))$.

Lemma 28. Let $f: W \rightarrow \mathbb{R}$ be Lipschitzian with constant $\ell$ on a ball $B(\bar{w}, \varrho)$ of $W$. Then for $\sigma \in] 0, \varrho[$ small enough and for any $w \in B(\bar{w}, \sigma)$ and any $w^{*} \in \partial^{C} f(w)$ one has $\left(w^{*},-1\right) \in \partial^{C} d_{E}(x)$, where $E$ is the epigraph 
of $f$ and $x:=(w, f(w)), X:=W \times \mathbb{R}$ being endowed with the norm given by $\|(w, r)\|=\ell\|w\|+|r|$.

Proof of the theorem in the general case. Since intrinsic $p$-paraconvexity is preserved when using an equivalent norm, we may use the norm described in the lemma and take $\varrho, \sigma>0$ as there. We use the implication (a) $\Rightarrow(\mathrm{c})$ of Corollary 21: there exist $c>0, \gamma \in] 0, \sigma\left[\operatorname{such}\right.$ that for any $x, x^{\prime} \in E \cap B(\bar{x}, \gamma)$ and $x^{*} \in \partial^{C} d_{E}(x)$,

$$
\left\langle x^{*}, x^{\prime}-x\right\rangle \leq c\left\|x-x^{\prime}\right\|^{p} .
$$

Let $\delta:=\gamma / 4 \ell$. Now, by the preceding lemma, for $w \in B(\bar{w}, \delta), u \in \delta \bar{B}_{W}$, $w^{\prime}:=w+u \in B(\bar{w}, 2 \delta)$ and $w^{*} \in \partial^{C} f(w)$ we have $x:=(w, f(w)) \in$ $E \cap B(\bar{x}, \gamma), x^{\prime}:=\left(w^{\prime}, f\left(w^{\prime}\right)\right) \in E \cap B(\bar{x}, \sigma)$ and $\left(w^{*},-1\right) \in \partial^{C} d_{E}(x)$. Then, by $(29)$,

$$
\left\langle w^{*}, u\right\rangle-\left(f\left(w^{\prime}\right)-f(w)\right) \leq c\left(\ell\left\|w^{\prime}-w\right\|+\left|f\left(w^{\prime}\right)-f(w)\right|\right)^{p} \leq c 2^{p} \ell^{p}\|u\|^{p} .
$$

Thus, for any $\varepsilon>0$, there exists $\delta>0$ such that for $w \in B(\bar{w}, \delta)$, any $w^{*} \in \partial^{C} f(w)$ and $u \in \bar{B}(0, \delta)$ one has $f(w+u)-f(w) \geq\left\langle w^{*}, u\right\rangle-c 2^{p} \ell^{p}\|u\|^{p}$, so that $f$ is $p$-paraconvex around $\bar{w}$ by Theorem 8 .

Finally, let us turn to sublevel sets.

Proposition 29. Let $X$ be an Asplund space and let $f: X \rightarrow \mathbb{R}_{\infty}$. Suppose $f$ is continuous and p-paraconvex around $\bar{x} \in S:=\{x \in X: f(x)$ $\leq 0\}$ and there exist $b, r>0$ such that $\left\|x^{*}\right\| \geq b$ for all $x \in(X \backslash S) \cap B(\bar{x}, r)$ and $x^{*} \in \partial^{F} f(x)$. Then $S$ is intrinsically p-paraconvex around $\bar{x}$.

Proof. Without loss of generality we may suppose $f$ takes the value $+\infty$ on $X \backslash U$, where $U:=B(\bar{x}, r)$. Then, by [74], [16], [29], [46, Thm. 9.1], [41, Thm. 3.2] and several other contributions, we have $f_{+}(x) \geq b d_{S}(x)$ for $x \in U$ for $f_{+}:=\max (f, 0)$. Let $\varepsilon>0$ be given. Using Theorem 8 , we can find $c>0$ and $\delta \in] 0, r[$ such that

$$
\left\langle x^{*}, x^{\prime}-x\right\rangle \leq f\left(x^{\prime}\right)-f(x)+c\left\|x^{\prime}-x\right\|^{p}
$$

for any $x, x^{\prime} \in B(\bar{x}, \delta)$ and $x^{*} \in \partial^{F} f(x)$. Given $x, x^{\prime} \in S \cap B(\bar{x}, \delta)$ and $x^{*} \in$ $\partial^{F} d_{S}(x)$, using [41, Cor. 4.1] we can find sequences $\left(\lambda_{n}\right),\left(x_{n}\right),\left(x_{n}^{*}\right)$ in $[0,1]$, $X, X^{*}$ respectively such that $\left(x_{n}\right) \rightarrow x,\left(\lambda_{n} x_{n}^{*}\right) \rightarrow x^{*}$ and $x_{n}^{*} \in \partial^{F} f\left(x_{n}\right)$ for each $n \in \mathbb{N}$. Suppose first $x^{*} \neq 0$. Then $f(x)=0$ since $f$ is continuous at $x$ and $x$ cannot belong to the interior of $S$. For $n$ so large that $x_{n} \in B(\bar{x}, \delta)$, and for $x^{\prime} \in S \cap B(\bar{x}, \delta)$, we have

$$
\left\langle x_{n}^{*}, x^{\prime}-x_{n}\right\rangle \leq f\left(x^{\prime}\right)-f\left(x_{n}\right)+c\left\|x^{\prime}-x_{n}\right\|^{p} .
$$

Thus

$$
\begin{aligned}
\left\langle x^{*}, x^{\prime}-x\right\rangle & =\lim _{n}\left\langle\lambda_{n} x_{n}^{*}, x^{\prime}-x_{n}\right\rangle \\
& \leq \limsup _{n} \lambda_{n}\left(f\left(x^{\prime}\right)-f\left(x_{n}\right)+c\left\|x^{\prime}-x_{n}\right\|^{p}\right) \leq c\left\|x^{\prime}-x\right\|^{p}
\end{aligned}
$$


since $f\left(x^{\prime}\right) \leq 0, \lambda_{n} \in[0,1]$ and $\left(f\left(x_{n}\right)\right) \rightarrow 0$. When $x^{*}=0$, the inequality $\left\langle x^{*}, x^{\prime}-x\right\rangle \leq c\left\|x^{\prime}-x\right\|^{p}$ is obvious. Thus, assertion (c) of Corollary 19 is satisfied for $E:=S$ and $S$ is intrinsically $p$-paraconvex around $\bar{x}$.

8. A counter-example. Because the concepts of paraconvexity and intrinsic paraconvexity seem to be so close, one may wonder whether they coincide. The following counter-example shows that this is not the case.

Given $p=3 / 2$, let $E$ be the hypograph of the function $f: r \mapsto|r|^{p}$ from $\mathbb{R}$ to $\mathbb{R}$ :

$$
E:=\left\{(r, s) \in \mathbb{R}^{2}: s \leq|r|^{p}\right\} .
$$

Let us endow $\mathbb{R}^{2}$ with the Euclidean norm and show that $E$ is intrinsically paraconvex around $(0,0)$, but not paraconvex around $(0,0)$. Since $E$ is the image under the isometry $(r, s) \mapsto(r,-s)$ of the epigraph of the function $-f$ whose derivative is $(p-1)$-Hölderian, it is intrinsically $p$-paraconvex around $(0,0)$. Let $(r, s) \in \mathbb{R}^{2}$ be close to $(0,0)$, with $r \geq 0, s>r^{p}$. In order to compute $d_{E}^{2}(r, s)$ we have to minimize the function $x \mapsto(x-r)^{2}+\left(x^{p}-s\right)^{2}$. The necessary condition is

$$
x-r+p x^{p-1}\left(x^{p}-s\right)=0 .
$$

Let $y:=x^{1 / 2}$. The preceding equation is equivalent to

$$
2 y^{2}-2 r+3 y\left(y^{3}-s\right)=0 .
$$

Fixing $s$, this equation defines $y:=x^{1 / 2}$ as an implicit function of $r$; moreover, $y(0)=y_{0}$, where $y_{0}>0$ is the unique solution of the equation $3 y^{3}+2 y-3 s=0$. The implicit function theorem asserts that $y^{\prime}(0)$ is determined by the relation

$$
y^{\prime}(0)\left[4 y_{0}+12 y_{0}^{3}-3 s\right]=2
$$

obtained by differentiating (31). Thus, since $x^{\prime}(0)=2 y^{\prime}(0) y_{0}$ and $3 s=$ $3 y_{0}^{3}+2 y_{0}$ by $(31)$,

$$
x^{\prime}(0)=\frac{4}{2+9 x(0)} .
$$

Therefore, for some function $r \mapsto q(r)$ with limit $x^{\prime}(0)$ as $r \rightarrow 0_{+}$, we have

$$
\begin{aligned}
d_{E}^{2}(r, s)= & \left(x_{0}+q(r) r-r\right)^{2}+\left(\left(x_{0}+q(r) r\right)^{3 / 2}-s\right)^{2} \\
= & x_{0}^{2}+2 x_{0}(q(r)-1) r+(q(r)-1)^{2} r^{2} \\
& +\left(x_{0}+q(r) r\right)^{3}-2 s\left(x_{0}+q(r) r\right)^{3 / 2}+s^{2} \\
= & \left(x_{0}^{2}+x_{0}^{3}-2 s x_{0}^{3 / 2}+s^{2}\right)+2 x_{0}(q(r)-1) r \\
& +3 x_{0}^{2} q(r) r-3 s x_{0}^{1 / 2} q(r) r+o(r)
\end{aligned}
$$


and since, by (30), $3 s x_{0}^{1 / 2}=3 x_{0}^{2}+2 x_{0}$, taking limits as $r \rightarrow 0$, we get

$$
\begin{aligned}
r^{-1}\left(d_{E}^{2}(r, s)-d_{E}^{2}(0, s)\right) & \rightarrow 2 x_{0}\left(x^{\prime}(0)-1\right)+3 x_{0}^{2} x^{\prime}(0)-3 s x_{0}^{1 / 2} x^{\prime}(0) \\
& =-2 x_{0} .
\end{aligned}
$$

Thus, given $a \in] 0,2 x_{0}[$, for $r>0$ small enough, we have

$$
d_{E}^{2}(r, s)-d_{E}^{2}(0, s) \leq-a r
$$

On the other hand, if $d_{E}$ is $p$-paraconvex, one can find $c, \delta>0$ such that for $\|(r, s)\| \leq \delta$,

$$
d_{E}(0, s) \leq \frac{1}{2} d(r, s)+\frac{1}{2} d(-r, s)+\frac{1}{4} c(2 r)^{p} .
$$

Now, since $E$ is invariant under the symmetry $(r, s) \mapsto(-r, s)$, we have $d_{E}(r, s) \geq d_{E}(0, s)-2^{p-2} c r^{p}$ and for $r$ sufficiently small the right hand side of this inequality is positive, so that

$$
\begin{aligned}
d_{E}^{2}(r, s) & \geq\left(d_{E}(0, s)-2^{p-2} c r^{p}\right)^{2}, \\
d_{E}^{2}(r, s)-d_{E}^{2}(0, s) & \geq-2^{p-1} c r^{p} d_{E}(0, s)+2^{2 p-4} c^{2} r^{2 p},
\end{aligned}
$$

a contradiction with $(32)$ when $\|(r, s)\|$ is sufficiently small.

9. Paraconvex sets and projections. The following result shows that, in the context of superreflexive spaces, $p$-paraconvexity of a distance function is equivalent to its continuous differentiability with a Hölderian derivative. It is reminiscent of $[14, \mathrm{Thm} .4 .8]$ which is set in a Hilbert space. There, $p=2$ and a Lipschitz property is given instead of a Hölderian property. Moreover, here $U$ is not a uniform enlargement of $E$; it may be small (or large) and far from $E$.

TheOREm 30. Let $X$ be a superreflexive Banach space, let $E$ be a closed subset of $X$ and let $U$ be an open subset of $X$. The following assertions relative to some choice of $p \in] 1,2]$ and of an equivalent norm on $X$ are equivalent:

(a) Each $w \in U$ has a unique metric projection $P_{E}(w)$ in $E$ and the mapping $w \mapsto P_{E}(w)$ is locally Hölderian on $U \backslash E$.

(b) $d_{E}(\cdot)$ is differentiable with a locally Hölderian derivative on $U \backslash E$.

(c) For each $\bar{w} \in U \backslash E, d_{E}(\cdot)$ is p-paraconvex around $\bar{w}$ for some $p \in$ ] $1,2]$.

Proof. (a) $\Rightarrow$ (b). As in [40, Thm. 21], for any $w \in U \backslash E$, we can show that $d_{E}$ is differentiable and

$$
d_{E}^{\prime}(w)=j\left(w-P_{E}(w)\right)
$$

where $j:=(\|\cdot\|)^{\prime}$. Let us prove that $d_{E}^{\prime}($.$) is locally (p-1)$-Hölderian on $U \backslash E$, taking on $X$ an equivalent norm of type $p$, i.e. such that its modulus 
of smoothness $\varrho_{X}$ satisfies (for some $k>0$ ) $\varrho_{X}(t) \leq k t^{p}$ for $t \in \mathbb{R}_{+}$, which is possible by [6, Thm. A.6]. Then by (15), for any $s>r>0$ there exists some $c>0$ such that

$$
\|j(x)-j(y)\| \leq c\|x-y\|^{p-1} \quad \forall x, y \in s \bar{B}_{X} \backslash r \bar{B}_{X} .
$$

Let $\bar{w} \in U \backslash E$ be given. By assumption, there exist $\gamma \in] 0,1]$ and $\ell, \delta>0$ such that

$$
\left\|P_{E}(u)-P_{E}(w)\right\| \leq \ell\|u-w\|^{\gamma} \quad \forall u, w \in B(\bar{w}, \delta) .
$$

By the above relations, using the fact that for $w$ close enough to $\bar{w}$ the number $\left\|w-P_{E}(w)\right\|$ lies in some interval $[r, s]$ with $s>r>0$, there exist $c, \varrho>0$ such that for all $u, w \in B(\bar{w}, \varrho)$,

$$
\begin{aligned}
\left\|d_{E}^{\prime}(u)-d_{E}^{\prime}(w)\right\| & =\left\|j\left(u-P_{E}(u)\right)-j\left(w-P_{E}(w)\right)\right\| \\
& \leq c\left\|\left(u-P_{E}(u)\right)-\left(w-P_{E}(w)\right)\right\|^{p-1} \\
& \leq c\left(\|u-w\|+\left\|P_{E}(u)-P_{E}(w)\right\|\right)^{p-1} \\
& \leq c\left(\|u-w\|+\ell\|u-w\|^{\gamma}\right)^{p-1} \\
& \leq c\left(2 \varrho^{1-\gamma}+\ell\right)^{p-1}\|u-w\|^{\gamma(p-1)} .
\end{aligned}
$$

(b) $\Rightarrow$ (c) is a consequence of Corollary 10 .

(c) $\Rightarrow$ (b). Since $X$ is superreflexive, it can be endowed with an equivalent uniformly smooth norm with a modulus of smoothness $\varrho_{X}$ satisfying for some $k>0$ and $p>1$ the estimate $\varrho_{X}(t) \leq k t^{p}$ for $t \geq 0$. By Corollary 13 , the function $-d_{E}$ is $p$-paraconvex. Thus, with the assumption of (c), $\partial^{F}\left(-d_{E}\right)(w) \neq \emptyset$ and $\partial^{F} d_{E}(w) \neq \emptyset$ at any $w \in U \backslash E$. Therefore, $d_{E}(\cdot)$ is Fréchet differentiable on $U \backslash E$.

Let us prove that $d_{E}^{\prime}(\cdot)$ is locally Hölderian on $U \backslash E$. Let $\bar{x} \in U \backslash E$ and assume that $d_{E}(\cdot)$ is $p$-paraconvex around $\bar{x}$. By the $p$-paraconvexity of $d_{E}(\cdot)$ and $-d_{E}(\cdot)$, by virtue of Theorem 8 , there exist $c, \delta>0$ such that for all $u, w \in B(\bar{x}, \delta), v \in \delta \bar{B}_{X}$,

$$
\begin{aligned}
-\left\langle d_{E}^{\prime}(u), v\right\rangle & \leq-d_{E}(u+v)+d_{E}(u)+c\|v\|^{p}, \\
\left\langle d_{E}^{\prime}(w), v\right\rangle & \leq d_{E}(w+v)-d_{E}(w)+c\|v\|^{p} .
\end{aligned}
$$

Thus, for any $u, w \in B(\bar{x}, \delta)$, for all $v \in B(0, \delta)$, we have

$$
\left\langle d_{E}^{\prime}(w)-d_{E}^{\prime}(u), v\right\rangle \leq 2\|u-w\|+2 c\|v\|^{p} .
$$

Hence

$$
\left\|d_{E}^{\prime}(u)-d_{E}^{\prime}(w)\right\| \leq \frac{2\|u-w\|}{t}+2 c t^{p-1}
$$

for all $t \in] 0, \delta\left[\right.$. Taking $\left.t=2^{-1 / p} \delta^{1-1 / p}\|u-w\|^{1 / p} \in\right] 0, \delta[$, we obtain 


$$
\begin{array}{r}
\left\|d_{E}^{\prime}(u)-d_{E}^{\prime}(w)\right\| \leq\left(2^{1+1 / p} \delta^{(1-p) / p}+2^{1 / p} \delta^{(p-1)^{2} / p} c\right)\|u-w\| \|^{(p-1) / p} \\
\forall u, w \in B(\bar{x}, \delta) .
\end{array}
$$

(b) $\Rightarrow($ a). By a result of Pisier (see [6, Thm. A.6], [52], [53]) there exists an equivalent norm on $X$ and $c>0, q \geq 2$ such that

$$
\delta_{X}(t):=\inf \left\{1-\frac{1}{2}\|x+y\|: x, y \in \bar{B}_{X},\|x-y\| \geq t\right\} \geq c t^{q} \quad \forall t \in[0,2] .
$$

Moreover, by [21, Prop. 5.2, p. 159] we may assume $\|\cdot\|$ is differentiable on $X \backslash\{0\}$. Now, by [70, Thm. 1], given $s>r>0$, one can find some $b>0$ such that for any $x, y \in s \bar{B}_{X} \backslash r \bar{B}_{X}$,

$$
\langle J(x)-J(y), x-y\rangle \geq b \delta_{X}(\|x-y\| / 2 s),
$$

where $J(\cdot):=\left(\frac{1}{2}\|\cdot\|^{2}\right)^{\prime}=\|\cdot\| j(\cdot)$. It follows that for such $x, y$,

$$
\|J(x)-J(y)\| \geq 2^{-q} s^{-q} b c\|x-y\|^{q-1} .
$$

By [40], the metric projection mapping $P_{E}(\cdot)$ is single-valued and continuous on $U \backslash E$. Furthermore, $d_{E}^{\prime}(w)=j\left(w-P_{E}(w)\right)$ for all $w \in U \backslash E$, hence $\left(\frac{1}{2} d_{E}^{2}\right)^{\prime}(w)=J\left(w-P_{E}(w)\right)$. Let us prove that $w \mapsto P_{E}(w)$ is locally Hölderian on $U \backslash E$. Since $d_{E}^{\prime}$ is locally Hölderian, $\left(\frac{1}{2} d_{E}^{2}\right)^{\prime}$ is also locally Hölderian as

$$
\left\|\left(d_{E}^{2}\right)^{\prime}(x)-\left(d_{E}^{2}\right)^{\prime}(y)\right\| \leq 2 d_{E}(x)\left\|d_{E}^{\prime}(x)-d_{E}^{\prime}(y)\right\|+2\left\|d_{E}^{\prime}(y)\right\|\|x-y\| .
$$

Given $\bar{x} \in U \backslash E$, assumption (b) yields some $\gamma \in] 0,1]$ and $a, \delta>0$ such that

$$
\left\|J\left(u-P_{E}(u)\right)-J\left(w-P_{E}(w)\right)\right\| \leq a\|u-w\|^{\gamma} \quad \forall u, w \in B(\bar{x}, \delta) .
$$

Then inequality (33) yields

$$
\left\|\left(u-P_{E}(u)\right)-\left(w-P_{E}(w)\right)\right\|^{q-1} \leq 2^{q} s^{q} b^{-1} c^{-1} a\|u-w\|^{\gamma},
$$

hence

$$
\begin{aligned}
\| P_{E}(u)- & P_{E}(w) \| \\
& \leq\|u-w\|+2^{q /(q-1)} s^{q /(q-1)} b^{1 /(1-q)} c^{1 /(1-q)} a^{1 /(q-1)}\|u-w\|^{\gamma /(q-1)} .
\end{aligned}
$$

This shows that the metric projection $P_{E}(\cdot)$ is locally Hölderian around $\bar{x}$.

Acknowledgements. The authors are grateful to C. Zălinescu for a careful reading of the manuscript and to an anonymous referee who pointed out some additional references and limitations. The first author would like to thank the Department of Mathematics of the University of Pau for hospitality and support. 


\section{References}

[1] Ya. I. Alber, A bound for the modulus of continuity for metric projections in a uniformly convex and uniformly smooth Banach space, J. Approx. Theory 85 (1996), 237-249.

[2] Ya. I. Alber and A. I. Notik, On some estimates for projection operators in Banach spaces, Comm. Appl. Nonlinear Anal. 2 (1995), 47-55.

[3] H. Attouch and D. Azé, Approximation and regularization of arbitrary functions in Hilbert spaces by the Lasry-Lions method, Ann. Inst. Henri Poincaré Anal. Non Linéaire 10 (1993), 289-312.

[4] D. Aussel, J.-N. Corvellec and M. Lassonde, Mean value property and subdifferential criteria for lower semicontinuous functions, Trans. Amer. Math. Soc. 347 (1995), 4147-4161.

[5] D. Aussel, A. Daniilidis and L. Thibault, Subsmooth sets: functional characterizations and related concepts, ibid. 357 (2005), 1275-1301.

[6] Y. Benyamini and J. Lindenstrauss, Geometric Nonlinear Functional Analysis, Amer. Math. Soc. Colloq. Publ. 48, Providence, 2000.

[7] J. M. Borwein and J. R. Giles, The proximal normal formula in Banach space, Trans. Amer. Math. Soc. 302 (1987), 371-381.

[8] M. L. Bougeard, Morse theory for some lower- $C^{2}$ functions in finite dimension, Math. Program. Ser. A 41 (1988), 141-159.

[9] M. Bougeard, J.-P. Penot and A. Pommellet, Towards minimal assumptions for the infimal convolution regularization, J. Approx. Theory 64 (1991), 245-270.

[10] A. Canino, On p-convex sets and geodesics, J. Differential Equations 75 (1988), 118-157.

[11] P. Cannarsa and C. Sinestrari, Semiconcave Functions, Hamilton-Jacobi Equations and Optimal Control, Birkhäuser, Basel, 2004.

[12] M. Castellani and M. Pappalardo, Characterization of @-convex functions, in: Generalized Convexity, Generalized Monotonicity: Recent Results, J.-P. Crouzeix et al. (eds.), Kluwer, Dordrecht, 1998, 219-233.

[13] F. H. Clarke, Optimization and Nonsmooth Analysis, Wiley Interscience, New York, 1983.

[14] F. H. Clarke, R. J. Stern and P. R. Wolenski, Proximal smoothness and the lower-C $C^{2}$ property, J. Convex Anal. 2 (1995), 117-144.

[15] G. Colombo and V. Goncharov, Variational inequalities and regularity properties of closed sets in Hilbert spaces, J. Convex Anal. 8 (2001), 197-221.

[16] O. Cornejo, A. Jourani and C. Zalinescu, Conditioning and upper-Lipschitz inverse subdifferentials in nonsmooth optimization problems, J. Optim. Theory Appl. 95 (1997), 127-148.

[17] M. G. Crandall and A. Pazy, Nonlinear evolution equations in Banach spaces, Israel J. Math. 11 (1972), 57-94.

[18] A. Daniilidis and P. Georgiev, Approximate convexity and submonotonicity, J. Math. Anal. Appl. 291 (2004), 292-301.

[19] E. De Giorgi, A. Marino and M. Tosques, $(p, q)$-convex functions, Atti Accad. Naz. Lincei Rend. Cl. Sci. Fis. Mat. Natur. 73 (1982), 6-14.

[20] M. Degiovanni, A. Marino and M. Tosques, General properties of $(p, q)$-convex functions and $(p, q)$-monotone operators, Ric. Mat. 32 (1983), 285-319.

[21] R. Deville, G. Godefroy and V. Zizler, Smoothness and Renormings in Banach Spaces, Pitman Monogr. Surveys Pure Appl. Math. 64, Longman, Harlow, 1993. 
[22] H. Federer, Curvature measures, Trans. Amer. Math. Soc. 93 (1959), 418-491.

[23] B. Ginsburg and A. D. Ioffe, The maximum principle in optimal control of systems governed by semilinear equations, in: Nonsmooth Analysis and Geometric Methods in Deterministic Optimal Control (Minneapolis, MN, 1993), B. S. Mordukhovich et al. (eds.), IMA Vol. Math. Appl. 78, Springer, New York, 1996, 81-110.

[24] J. W. Green, Approximately convex functions, Duke Math. J. 19 (1952), 499-504.

[25] D. H. Hyers and S. M. Ulam, Approximately convex functions, Proc. Amer. Math. Soc. 3 (1952), 821-828.

[26] A. D. Ioffe, Approximate subdifferentials and applications. III: The metric theory, Mathematika 36 (1989), 1-38.

[27] — , Proximal analysis and approximate subdifferentials, J. London Math. Soc. 41 (1990), 175-192.

[28] —, Fuzzy principles and characterization of trustworthiness, Set-Valued Anal. 6 (1998), 265-276.

[29] - Metric regularity and subdifferential calculus, Uspekhi Mat. Nauk 55 (2000), no. 3, 103-162 (in Russian); English transl.: Russian Math. Surveys 55 (2000), $501-558$.

[30] A. Jourani, Subdifferentiability and subdifferential monotonicity of $\gamma$-paraconvex functions, Control Cybernet. 25 (1996), 721-737.

[31] A. Y. Kruger and B. S. Mordukhovich, Extremal points and the Euler equation in nonsmooth optimization problems, Dokl. Akad. Nauk BSSR 24 (1980), 684-687.

[32] J.-M. Lasry and P.-L. Lions, A remark on regularization in Hilbert spaces, Israel J. Math. 55 (1986), 257-266.

[33] G. Lebourg, Valeur moyenne pour gradient généralisé, C. R. Acad. Sci. Paris Sér. A-B 281 (1975), 795-797.

[34] Ph. D. Loewen, A mean value theorem for Fréchet subgradients, Nonlinear Anal. 23 (1994), 1365-1381.

[35] E. Michael, Paraconvex sets, Math. Scand. 7 (1959), 372-376.

[36] Ph. Michel and J.-P. Penot, A generalized derivative for calm and stable functions, Differential Integral Equations 5 (1992), 433-454.

[37] B. S. Mordukhovich and Y. Shao, Nonsmooth sequential analysis in Asplund spaces, Trans. Amer. Math. Soc. 348 (1996), 1235-1280.

[38] H. V. Ngai, D. T. Luc and M. Théra, Approximate convex functions, J. Nonlinear Convex Anal. 1 (2000), 155-176.

[39] H. V. Ngai and J.-P. Penot, Approximately convex functions and approximately monotone operators, Nonlinear Anal. 66 (2007), 547-564.

[40] - - -, Approximately convex sets, J. Nonlinear Convex Anal. 8 (2007), no. 3, to appear.

[41] H. V. Ngai and M. Théra, Metric inequality, subdifferential calculus and applications, Set-Valued Anal. 9 (2001), 187-216.

[42] A. Pazy, Semi-groups of nonlinear contractions in Hilbert space, in: Problems in Non-Linear Analysis (Varenna, 1970), Ed. Cremonese, 1971, 343-430.

[43] - On the asymptotic behavior of semigroups of nonlinear contractions in Hilbert space, J. Funct. Anal. 27 (1978), 292-307.

[44] J.-P. Penot, On the Mean Value Theorem, Optimization 19 (1988), 147-156.

[45] - Favorable classes of mappings and multimappings in nonlinear analysis and optimization, J. Convex Anal. 3 (1996), 97-116.

[46] —, Well-behavior, well-posedness and nonsmooth analysis, Pliska Stud. Math. Bulgar. 12 (1998), 141-190. 
[47] J.-P. Penot, Miscellaneous incidences of convergence theories in optimization and nonlinear analysis. II: Applications in nonsmooth analysis, in: Recent Advances in Nonsmooth Optimization, D. Z. Du et al. (eds.), World Sci., Singapore, 1995, $289-321$.

[48] —, The compatibility with order of some subdifferentials, Positivity 6 (2002), 413432.

[49] - Differentiability properties of optimal value functions, Canad. J. Math. 56 (2004), 825-842.

[50] J.-P. Penot and R. Ratsimahalo, Uniform continuity of projection mappings in Banach spaces, Comm. Appl. Nonlinear Anal. 6 (1999), 1-17.

[51] J.-P. Penot and M. Volle, On strongly convex and paraconvex dualities, in: Generalized Convexity and Fractional Programming with Economic Applications (Pisa, 1988), Lecture Notes in Econom. Math. Systems 345, Springer, 1990, 198-218.

[52] G. Pisier, Martingales with values in uniformly convex spaces, Israel J. Math. 20 (1975), 326-350.

[53] B. Prus and R. Smarzewski, Strongly unique best approximations and centers in uniformly convex spaces, J. Math. Anal. Appl. 121 (1987), 10-21.

[54] R. T. Rockafellar, Favorable classes of Lipschitz-continuous functions in subgradient optimization, in: Progress in Nondifferentiable Optimization, E. Nurminski (ed.), Pergamon Press, New York.

[55] R. T. Rockafellar and R. J.-B. Wets, Variational Analysis, Springer, New York, 1998.

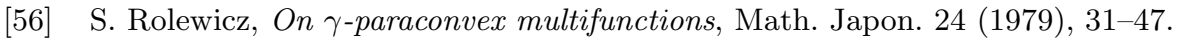

[57] - On $\alpha(\cdot)$-monotone multifunctions and differentiability of $\gamma$-paraconvex functions, Studia Math. 133 (1999), 29-37.

[58] - On $\alpha(\cdot)$-paraconvex and strongly $\alpha(\cdot)$-paraconvex functions, Control Cybernet. 29 (2000), 367-377.

[59] - On uniformly approximate convex and strongly $\alpha(\cdot)$-paraconvex functions, ibid. 30 (2001), 323-330.

[60] - On the coincidence of some subdifferentials in the class of $\alpha(\cdot)$-paraconvex functions, Optimization 50 (2001), 353-360.

[61] -, On $\alpha(\cdot)$-monotone multifunctions and differentiability of strongly $\alpha(\cdot)$-paraconvex functions, Control Cybernet. 31 (2002), 601-619.

[62] -, Paraconvex analysis, ibid. 34 (2005), 951-965.

[63] - On differentiability of strongly $\alpha(\cdot)$-paraconvex functions in non-separable Asplund spaces, Studia Math. 167 (2005), 235-244.

[64] -, Paraconvex analysis on $C_{E}^{1, u}$-manifolds, Optimization 55 (2006), 49-60.

[65] J. E. Spingarn, Submonotone subdifferentials of Lipschitz functions, Trans. Amer. Math. Soc. 264 (1981), 77-89.

[66] L. Thibault and D. Zagrodny, Integration of subdifferentials of lower semicontinuous functions on Banach spaces, J. Math. Anal. Appl. 189 (1995), 33-58.

[67] -, - Enlarged inclusion of subdifferentials, Canad. Math. Bull. 48 (2005), 283-301.

[68] J. Treiman, Shrinking generalized gradients, Nonlinear Anal. 12 (1988), 1429-1450.

[69] J.-Ph. Vial, Strong and weak convexity of sets and functions, Math. Oper. Res. 8 (1983), 231-259.

[70] Z.-B. Xu and G. F. Roach, Characteristic inequalities of uniformly convex and uniformly smooth Banach spaces, J. Math. Anal. Appl. 157 (1991), 189-210.

[71] D. Zagrodny, Approximative mean value theorem for upper subderivatives, Nonlinear Anal. 12 (1988), 1413-1428. 
[72] D. Zagrodny, A note on the equivalence between the mean value theorem for the Dini derivative and the Clarke-Rockafellar derivative, Optimization 21 (1990), 179-183.

[73] C. Zalinescu, Convex Analysis in General Vector Spaces, World Sci., Singapore, 2002.

[74] R. Zhang and J. Treiman, Upper-Lipschitz multifunctions and inverse subdifferentials, Nonlinear Anal. 24 (1995), 273-286.

Department of Mathematics

Pedagogical University of Quynhon

170 An Duong Vuong

Qui Nhon, Vietnam

E-mail: ngaivn@yahoo.com
Laboratoire de Mathématiques Appliquées

CNRS UMR 5142

Faculté des Sciences

Av. de l'Université

64000 Pau, France

E-mail: jean-paul.penot@univ-pau.fr

Received July 1, 2006

Revised version August 2, 2007 